Named Series: Blood-Brain Interfaces

\title{
The choroid plexus epithelium as a novel player in the stomach-brain axis during Helicobacter infection
}

\section{A R T I C L E I N F O}

\section{Article history:}

Received 10 July 2017

Received in revised form 11 December 2017

Accepted 15 December 2017

Available online 16 December 2017

\section{Keywords:}

Helicobacter

Stomach-brain axis

Gut-brain axis

Choroid plexus

Blood-brain barrier

\begin{abstract}
A B S T R A C T
Several studies suggest a link between shifts in gut microbiota and neurological disorders. Recently, we reported a high prevalence of Helicobacter suis (H. suis) in patients with Parkinson's disease. Here, we evaluated the effect of gastric $H$. suis infection on the brain in mice. One month of infection with $H$. suis resulted in increased brain inflammation, reflected in activation of microglia and cognitive decline. Additionally, we detected choroid plexus inflammation and disruption of the epithelial bloodcerebrospinal fluid (CSF) barrier upon $H$. suis infection, while the endothelial blood-brain barrier (BBB) remained functional. These changes were accompanied by leakage of the gastrointestinal barrier and low-grade systemic inflammation, suggesting that $H$. suis-evoked gastrointestinal permeability and subsequent peripheral inflammation induces changes in brain homeostasis via changes in blood-CSF barrier integrity. In conclusion, this study shows for the first time that $H$. suis infection induces inflammation in the brain associated with cognitive decline and that the choroid plexus is a novel player in the stomachbrain axis.
\end{abstract}

(c) 2017 Elsevier Inc. All rights reserved.

\section{Introduction}

The healthy human gastrointestinal tract harbours approximately 100 trillion commensal bacteria (Clemente et al., 2012; Foster and McVey Neufeld, 2013). An increasing number of recent studies indicate that shifts in the gastrointestinal microbiome caused by a broad variety of triggers, including gastrointestinal infections, might have a significant effect on our health. Indeed, dysbiosis has been associated with a wide range of human health problems (Boulange et al., 2016; Clemente et al., 2012; Dinan and Cryan, 2017b; Ochoa-Reparaz and Kasper, 2014; Walters et al., 2014; Zhang et al., 2015), including neurological disorders such as depression, anxiety-like behaviour, autism, Alzheimer's disease and Parkinson's disease (Bercik et al., 2011; Bravo et al., 2011; Clapp et al., 2017; Dinan and Cryan, 2017a; Fung et al.,

\footnotetext{
* Corresponding author at: VIB - Ghent University, FSVM Building, Technologiepark 927, B-9052 Zwijnaarde (Ghent), Belgium.

E-mail address: Roosmarijn.Vandenbroucke@irc.VIB-UGent.be (R.E. Vandenbroucke).

1 Equal contribution.
}

2017; Hu et al., 2016; Kelly et al., 2017; Mulak and Bonaz, 2015; Parracho et al., 2005; Tognini, 2017; Zhang et al., 2015).

The genus Helicobacter is a group of gram-negative, spiralshaped bacteria colonizing the gastrointestinal tract of humans and animals. Helicobacter pylori (H. pylori) is the best-known gastric human pathogen and is one of the major causes of gastric cancer (Kusters et al., 2006). Besides H. pylori, other Helicobacter species, referred to as non-Helicobacter pylori Helicobacter (NHPH) species, have also been associated with severe human gastric malignancies (Haesebrouck et al., 2009). The most common NHPH species in humans is H. suis (Haesebrouck et al., 2009), which naturally colonizes the stomach of pigs (Liang et al., 2013) and non-human primates (Bosschem et al., 2017a). Most likely, transmission of $H$. suis occurs through direct or indirect contact between animals and humans. Additionally, $H$. suis can be present and survive in minced pork, suggesting that raw or undercooked pork may be a source of $H$. suis infection in humans (De Cooman et al., 2013).

Recently, an exceptionally high frequency (27\%) of $H$. suis DNA was found in gastric biopsies from human patients with Parkinson's disease compared to a control group with no clinical 
parkinsonism (2\%) (Blaecher et al., 2013). Besides, H. suis DNA was also detected in a blood sample from a patient with both parkinsonism and Alzheimer's disease (Blaecher et al., 2013). This patient received triple therapy with tetracycline, clarithromycin and a proton pump inhibitor to eradicate $H$. suis. Interestingly, the gastric and neurological symptoms improved remarkably (Dobbs et al., 2013). This finding suggests that the inflammatory effects caused by Helicobacter are not restricted to the stomach.

To prevent disturbance of brain homeostasis due to alterations in the systemic blood composition, the brain is protected by a series of barriers, including the blood-brain barrier (BBB) and the blood-cerebrospinal fluid (CSF) barrier (De Bock et al., 2014). It has been suggested that systemic inflammation may contribute to the impairment of the BBB (Kortekaas et al., 2005; Stolp and Dziegielewska, 2009) and the blood-CSF barrier (Demeestere et al., 2015; Marques et al., 2009, 2007; Vandenbroucke et al., 2012). Furthermore, multiple neurodegenerative disorders, including Parkinson's disease and Alzheimer's disease, have been associated with disruption of the BBB (Lee and Pienaar, 2014; Li et al., 2015; Stolp and Dziegielewska, 2009) and the blood-CSF barrier (Balusu et al., 2016a; Bergen et al., 2015; Brkic et al., 2015; Gorle et al., 2016; Pisani et al., 2012; Vandenbroucke, 2016; Vawter et al., 1996). Because it has been proposed that Helicobacter infections cause systemic inflammation (Alvarez-Arellano and Maldonado-Bernal, 2014; Blaecher et al., 2013), gastric infection with $H$. suis could influence the integrity of the brain barriers and thereby contribute to the pathophysiology of neurodegenerative disorders.

In this study, we investigated the effect of gastric $H$. suis infection on brain homeostasis. Our results show that $H$. suis induces inflammation not only in the stomach, but also in the brain. We identified a novel role for the choroid plexus in the communication between the gastrointestinal tract and the brain: leakage of the gastrointestinal barrier induces low-grade systemic inflammation and subsequent loss of the blood-CSF barrier integrity, increased brain inflammation and cognitive decline.

\section{Materials and methods}

\subsection{Mice}

For the Helicobacter infection experiments, female C57BL/ 6JOlaHsd mice were purchased from Harlan (Harlan Laboratories, The Netherlands). The animals were housed in filtertop cages in groups of 5-10 mice/cage with free access to food and water and with a 12-h light/12-h dark cycle in a conventional animal house. All experiments were approved by the ethical committee of the Faculty of Veterinary Medicine of Ghent University.

\subsection{Cultivation of $H$. suis bacteria}

H. suis bacteria (strain HS1) were grown biphasically on $1.5 \%$ Brucella agar (Immunosource, Belgium), supplemented with $1 \mathrm{ml}$ skirrow (Oxoid, United Kingdom), $3 \mathrm{ml}$ vitox (Oxoid, United Kingdom), $5 \mu \mathrm{g} / \mathrm{ml}$ amphotericin B (Sigma-Aldrich, Germany), 0.05\% $\mathrm{HCl}$ (Sigma-Aldrich, Germany) and 20\% inactivated fetal bovine serum (Perbio, Thermo Scientific Hyclone, United Kingdom) with on top Brucella broth (Immunosource, Belgium), supplemented with $0.05 \% \mathrm{HCl}$ (Sigma-Aldrich, Germany) (Baele et al., 2008). The plates were incubated at $37^{\circ} \mathrm{C}$ under micro-aerobic conditions $\left(10 \% \mathrm{CO}_{2}, 5 \% \mathrm{O}_{2}\right.$ and $85 \% \mathrm{~N}_{2}$ ). Using a counting chamber (Neubauwer Improved Assistant) the bacteria were counted and diluted to $10^{8}$ viable $H$. suis bacteria $/ \mathrm{ml}$.

\subsection{In vivo infection procedure}

At the age of 6 weeks, animals were anaesthetized with 2.5\% isoflurane and intragastrically inoculated with either $300 \mu \mathrm{l}$ of a stock solution containing $10^{8}$ viable $H$. suis bacteria/ml or with $300 \mu \mathrm{l}$ of the growth medium of the bacteria (control group). One month after infection, mice were euthanized for further analysis by the administration of an overdose of ketamine/xylazine followed by decapitation.

\subsection{Tissue collection}

For RNA and protein analysis, mice were transcardially perfused with D-PBS/heparin ( $0.2 \%$ heparin) supplemented with $0.5 \%$ bromophenol blue. The stomach (including a small part of the duodenum) was collected and cut open via the curvatura major. After rinsing with sterile Hank's Buffered Salt Solution (Gibco by Thermo Fisher Scientific, USA), samples from the antrum and the corpus of the stomach and duodenum were collected and stored in RNAlater (Qiagen) at $-70^{\circ} \mathrm{C}$ or snap frozen in liquid nitrogen for RNA and protein extraction, respectively. Brain tissue was dissected out. The choroid plexus from all four ventricles was collected and snap frozen in liquid nitrogen, while hippocampus samples were stored in RNAlater (Qiagen) for RNA analysis or snap frozen in liquid nitrogen for DNA analysis.

For immunohistochemical analyses, mice were transcardially perfused with $4 \%$ paraformaldehyde (PFA). Brain tissue and samples from the antrum and corpus of the stomach and duodenum were collected. The brain was divided into the left and right hemisphere. The right hemisphere was successively fixated in 4\% PFA and embedded in paraffin for paraffin sections. In parallel, the left hemisphere was embedded in cryoprotectant (Thermo Scientific ${ }^{\mathrm{TM}}$ ), underwent rapid freezing on dry ice and was stored at $-70{ }^{\circ} \mathrm{C}$. The stomach and duodenum samples were either stored overnight in $20 \%$ sucrose, embedded in cryoprotectant (Thermo Scientific ${ }^{\mathrm{TM}}$ ), underwent rapid freezing on dry ice and were stored at $-70^{\circ} \mathrm{C}$ or were fixated overnight in a solution of $3.7 \%$ formaldehyde (Chem-lab) and subsequently embedded in paraffin (Labo-nord, VWR).

2.5. DNA extraction and quantification of colonizing $H$. suis bacteria in the stomach, duodenum and hippocampus

Stomach and duodenum samples stored in RNAlater were homogenized using the MagNAlyser (Roche diagnostics) and RNA and DNA were separated as described before (Joosten et al., 2013). DNA of the hippocampus was extracted with the Isolate II Genomic DNA kit (Bioline) according to the manufacturer's instructions. The number of colonizing $H$. suis bacteria per mg tissue was determined in the obtained DNA samples using Real-Time (RT)quantitative (q) Polymerase Chain Reaction (PCR). RT-qPCR detection of $H$. suis DNA was performed using the ureA-based primers (Table 1) (Blaecher et al., 2013) and the standard was generated as described before (O'Rourke et al., 2004). Standard and samples of stomach were run in duplicate on a CFX384 ${ }^{\mathrm{TM}} \mathrm{RT}-\mathrm{qPCR}$ System with a C1000 Thermal Cycler (Bio-Rad) according to the manufacturer's instructions. RT-qPCR for hippocampus samples was performed on the Light Cycler 480 system (Roche) using the $2 x$ Sensimix (Sybr No-Rox kit; Bioline) according to manufacturer's instructions.

\subsection{RNA extraction and RT-qPCR for gene expression}

Using the RNeasy mini kit (Qiagen), total RNA was extracted from the antrum and the corpus of the stomach, the duodenum, hippocampus and choroid plexus according to the manufacturer's 
Table 1

Overview of used qRT-PCR primer sequences.

\begin{tabular}{|c|c|c|}
\hline & Forward primer sequence & Reverse primer sequence \\
\hline H2afz & GGTATCACCCCTCGTCACTT & TCAGCGATTTGTGGATGTGT \\
\hline Hprt (stomach) & CAGGCCAGACTTTGTTGGAT & TTGCGCTCATCTTAGGCTTT \\
\hline Ppia & AGCATACAGGTCCTGGCATC & TTCACCTTCCCAAAGACCAC \\
\hline Il1 $\beta$ & CACCTCACAAGCAGAGCACAAG & GCATTAGAAACAGTCCAGCCCATAC \\
\hline$K c$ & GCTGGGATTCACCTCAAGAA & TCTCCGTTACTTGGGGACAC \\
\hline Mip2 & TGCCTGAAGACCCTGCCAAGG & GTTAGCCTTGCCTTTGTTCAG \\
\hline Lix & CTCAGTCATAGCCGCAACCGAGC & CCGTTCTTTCCACTGCGAGTGC \\
\hline Ocln & CCAGGCAGCGTGTTCCT & TTCTAAATAACAGTCACCTGAGGGC \\
\hline Cldn1 & TCTACGAGGGACTGTGGATG & TCAGATTCAGCAAGGAGTCG \\
\hline Cldn5 & GCAAGGTGTATGAATCTGTGCT & GTCAAGGTAACAAAGAGTGCCA \\
\hline Muc5ac & Purchased from Qiagen (Qt01196006) & \\
\hline Muc5b & CAGATCCATCCATCCCATTTCT & TATCTGACTACCACTTGTTGATGTGACT \\
\hline Muc6 & TGCTCCCAGAATGAGTACTTCGA & CAGAGGTGGAACTGTGAAACTCAGT \\
\hline Muc13 & GCCAGTCCTCCCACCACGGTA & CTGGGACCTGTGCTTCCACCG \\
\hline Tnf & ACCCTGGTATGAGCCCATATAC & ACACCСАTTCССТTCACAGAG \\
\hline iNos & CAGCTGGGCTGTACAAACCTT & CATTGGAAGTGAAGCGTTTCG \\
\hline Zo1 & AGGACACCAAAGCATGTGAG & GGCATTCCTGCTGGTTACA \\
\hline Zo3 & ACCCTATGGCCTGGGCTTC & CCCGGGTACAACGTGTCC \\
\hline Hprt (choroid plexus) & AGTGTTGGATACAGGCCAGAC & CGTGATTCAAATCCCTGAAGT \\
\hline Rpl & CCTGCTGCTCTCAAGGTT & TGGTTGTCACTGCCTGGTACTT \\
\hline$U b c$ & AGGTCAAACAGGAAGACAGACGTA & TCACACCCAAGAACAAGCACA \\
\hline Gapdh & TGAAGCAGGCATCTGAGGG & CGAAGGTGGAAGAGTGGGAG \\
\hline H. suis & AAAACAMAGGCGATCGCCCTGTA & TTTCTTCGCCAGGTTCAAAGCG \\
\hline
\end{tabular}

instructions and measured using Nanodrop equipment (Nanodrop ND-1000, Fisher Scientifics). cDNA was synthesized using a cDNA Synthesis Kit (Bio-Rad). RT-qPCR for analysis of the stomach and duodenum samples was performed on a CFX384 ${ }^{\mathrm{TM}}$ RT-qPCR System with a C1000 Thermal Cycler (Bio-Rad, Hercules), using the 2xSensimix (Sybr No-Rox kit; Bioline), while for brain samples the RTqPCR was performed on the Light Cycler 480 system (Roche) using the 2xSensimix (Sybr No-Rox kit; Bioline). Expression levels were normalized to the expression of 3 reference genes: Hprt, H2afz and Ppia for stomach and duodenum samples, Hprt, Rpl and $U b c$ for choroid plexus samples and Gapdh, Rpl and Ubc for hippocampus samples. The primer sequences are shown in Table 1.

\subsection{Cytokine/chemokine measurements}

The snap frozen gastrointestinal samples were lysed using lysis buffer containing 0.5\% CHAPS (Sigma-Aldrich) and a protease inhibitor complete tablet (Roche Applied Science; 11873580 001). Protein concentration of the samples was measured using the Pierce BCA protein assay kit (Thermofisher Scientific). Cytokines and chemokines in serum, CSF and protein lysates were measured using the Bio-Plex cytokine assays (Bio-Rad) according to the manufacturer's instructions.

\subsection{TLR4 activation analysis}

Serum levels of TLR4 in serum were measured using the HEKBlue mTLR4 assay (InvivoGen) according to the manufacturer's instructions. HEK-Blue mTLR4 cells in detection medium were seeded at 25.000 cells per well in a 96 -well plate. After $18 \mathrm{~h}$ incubation at $37{ }^{\circ} \mathrm{C}$ with 10 times diluted serum derived from control or $H$. suis infected mice, absorption of the culture medium was measured at $655 \mathrm{~nm}$ with a absorbance plate reader (iMark Microplate Absorbance Reader, Bio-Rad) and relative TLR4 activation was calculated.

\subsection{Quantification of the gastrointestinal permeability}

Gastrointestinal permeability was determined as previously described (Van Hauwermeiren et al., 2015; Vandenbroucke et al., 2014). Briefly, 4 kDa FITC-dextran (Sigma) was intragastrically administered five hours before collection of blood. Blood was collected in EDTA tubes (Sarstedt) by means of heart punction followed by centrifugation. Subsequently, plasma was isolated and diluted twice in sterile D-PBS. Gastrointestinal leakage was determined by measurement of fluorescence at $\lambda_{\text {ex }} / \lambda_{\text {em }}=485 / 520 \mathrm{~nm}$.

\subsection{Quantification of blood-CSF barrier and BBB permeability}

Blood-CSF barrier and BBB permeability were determined as previously described (Brkic et al., 2015; Vandenbroucke et al., 2012). Briefly, $4 \mathrm{kDa}$ FITC-dextran (Sigma) was injected iv $1 \mathrm{~h}$ before CSF collection. CSF was obtained from the fourth ventricle using the cisterna magna puncture method (Balusu et al., 2016b; Brkic et al., 2015; Vandenbroucke et al., 2012). Subsequently, mice were perfused with D-PBS/heparin (0.2\% heparin) and brain tissue was isolated. CSF samples were diluted 100-fold in sterile D-PBS, and blood-CSF barrier leakage was determined by measurement of fluorescence at $\lambda_{\mathrm{ex}} / \lambda_{\mathrm{em}}=485 / 520 \mathrm{~nm}$. Brain samples were cut into small pieces, incubated overnight at $37{ }^{\circ} \mathrm{C}$ in formamide while shaking and supernatant was collected after centrifugation for 15 min at maximum speed. Brain fluid was diluted 2-fold in sterile DPBS, and BBB leakage was determined by measurement of fluorescence at $\lambda_{\text {ex }} / \lambda_{\text {em }}=485 / 520 \mathrm{~nm}$.

\subsection{Histopathology and immunohistochemistry}

In order to evaluate inflammation in the stomach and duodenum, paraffin sections of $5 \mu \mathrm{m}$ were cut using a microtome (Microm, Prosan, Thermo-Fisher). Sections were stained with Hematoxylin Gill III Prosan (Merck) and Eosine Yellow (VWR) according to standardized protocols. The H\&E stainings of the stomach were applied to determine the grade of overall gastritis, using the modified visual analog scale similar to the adapted Updated Sydney System as previously described (Flahou et al., 2010). Gastric tissue was visualized using a light microscope (Leica DM2000).

For zonula occludens 1 (ZO1) immunostaining of the stomach and brain, $30 \mu \mathrm{m}$ cryosections and $20 \mu \mathrm{m}$ cryosections respectively were cut using a cryostat (Micron HM500) and mounted on slides. After air drying for $1 \mathrm{~h}$, sections were fixated with $1 \%$ PFA for 10 min for the stomach, while for the brain antigen retrieval was 
performed in citrate buffer (Dako; S2031). After a wash step for 5 min in PBS, sections were permeabilized for 10 min with $0.1 \%$ NP40. After two rinses with PBS, samples were blocked for $1 \mathrm{~h}$ at room temperature with $5 \%$ goat serum and then incubated with 1/500 diluted anti-ZO1 primary antibody (Invitrogen; 617300). After incubation overnight at $4{ }^{\circ} \mathrm{C}$, they were washed with PBS-T (PBS supplemented with $0.1 \%$ Tween 20 ) and $1 / 300$ diluted goat anti-rabbit DyLight633 secondary antibody (Thermo Scientific; 35562 ) diluted in $5 \%$ goat serum for $90 \mathrm{~min}$ at room temperature. Finally, a Hoechst staining was performed to counterstain the nuclei, the slides were dehydrated and xylene-based mounting media was applied. Cells were visualized using a confocal microscope (Leica TCS SP5 II).

For the MUC13 staining of the stomach sections of $5 \mu \mathrm{m}$ were cut from paraffin embedded tissue, using a microtome (Microm, Prosan, Thermo-Fisher), and placed on a microscope slide. Deparaffinization was performed by the addition of successively, xylene for $10 \mathrm{~min}$, isopropyl alcohol for $5 \mathrm{~min}$, 95\% ethanol for $5 \mathrm{~min}$, $50 \%$ ethanol for $5 \mathrm{~min}$ and aqua destillata for $5 \mathrm{~min}$. Antigen retrieval was performed in citrate buffer $(10 \mathrm{mM}$, pH6) using a pressure cooker, where after samples were rinsed with washing buffer (Dako). Slides were blocked for $5 \mathrm{~min}$ with peroxidase blocking reagent (Dako) and rinsed with aqua destillata and washing buffer (Dako). Successively slides were incubated for 30 min with the primary in-house polyclonal mouse MUC13 antibody, diluted in antibody diluent (1/1000, Dako, S3022). After a rinse step with washing buffer (Dako), samples were incubated with labelled polymer-HRP anti-mouse for $30 \mathrm{~min}$ at room temperature (Envi sion + system-HRP (DAB); K4007). Samples were rinsed 2 times with washing buffer (Dako), where after DAB + substrate (Dako) and $\mathrm{DAB}+$ chromogen (Dako) were added during 5 min. Finally, a counterstaining with haematoxylin was performed during 10 min, followed by a rinse step of 1 min using running tap water and a dehydration step. Gastric tissue was visualized using a light microscope (Leica DM2000).

For Helicobacter staining of the brain, $5 \mu \mathrm{m}$ sections were cut from paraffin embedded tissue and placed on a microscope slide. After deparaffinization and hydration, antigen retrieval was performed in citrate buffer $(10 \mathrm{mM}$, pH6) using a pressure cooker. Slides were incubated with $\mathrm{H}_{2} \mathrm{O}_{2}$ (Agilent; K4011). Successively, slides were rinsed and incubated for $30 \mathrm{~min}$ with a polyclonal rabbit anti-Helicobacter antibody (1/320) (Agilent; B0471). After rinsing, slides were incubated with Envision Link Rabbit (Agilent; K4011) and visualization was done using DAB chromogen (Agilent; K4011). Finally, a counterstaining with haematoxylin was performed and slides were dehydrated. Brain tissue was visualizes using Olympus BX51 microscope.

For IBA1 immunostaining, paraffin sections of the brains were processed as described before using the anti-IBA1 antibody (Wako; 019-19741) (Brkic et al., 2015). Imaging of whole brain sections was done using Zeiss Axio Scan.Z1 followed by quantification using Fiji (http://fiji.sc/Fiji). Quantification of the percentage of brown colour was done via colour thresholding, with correction for the total tissue surface, while the number of microglia $/ \mathrm{mm}^{2}$ were counted using the ZEN software.

For Claudin 1 (CLDN1) and GFAP immunostaining, paraffinembedded brains and stomach were sectioned at $5 \mu \mathrm{m}$, dewaxed, and rinsed in water and PBS before staining. Antigen retrieval was done using citrate buffer (Dako; S2031), followed by washing in PBS. For CLDN1 staining, peroxidase was blocked by incubating the slides in $3 \% \mathrm{H}_{2} \mathrm{O}_{2}$ in methanol for 10 min, followed by washing in PBS. Samples for CLDN1 and GFAP were blocked with 5\% goat serum in antibody diluent (Dako; S2022) for $30 \mathrm{~min}$ at RT. This was followed by incubation overnight at $4{ }^{\circ} \mathrm{C}$ with primary antibody (CLDN1, Invitrogen, 51-9000, 1/100; GFAP, DAKO, Z0334, 1/10000). The next day, slides were washed with PBS. For CLDN1 immunostaining, slides were incubated with secondary antibody coupled to HRP (Dako; E-K4003), and washed with PBS. An amplification step was performed using tyramide (TSA kit, Perkin Elmer) and visualization was done using $\mathrm{ABC}$ (Vector) and $\mathrm{DAB}$. Slides were counterstained with haematoxylin, dehydrated and xylene-based mounting media was applied. Cells were visualized using Olympus BX51 microscope. For GFAP, slides were incubated with $1 / 300$ diluted goat anti-rabbit-DyLight633 secondary antibody (Thermo Scientific; 35562 ) diluted in 5\% normal goat serum for $90 \mathrm{~min}$ at room temperature. Finally, a Hoechst staining was performed to counterstain the nuclei, the slides were dehydrated and xylenebased mounting media was applied. GFAP staining was visualized using Zeiss Axio Scan.Z1 followed by quantification using QuPath. Quantification of the percentage of positive red signal was done via colour thresholding, with correction for the total tissue surface. Representative images were taken using Leica TCS SP5 II.

\subsection{Western blot}

For CLDN1 and OCLN western blot analysis, choroid plexus tissue from two mice was pooled and homogenized in $50 \mu$ lysis buffer containing 0.5\% CHAPS and protease inhibitor complete tablet (Roche Applied Science; 11873580 001). Protein concentration was determined using the Thermo Scientific ${ }^{\mathrm{TM}}$ Pierce $^{\mathrm{TM}}$ Micro BCA ${ }^{\mathrm{TM}}$ Protein assay, and $80 \mu \mathrm{g}$ of protein was loaded on a 15\% SDS-PAGE gel. Wet blotting was performed overnight at constant current (90 $\mathrm{mA}$ ) at $4{ }^{\circ} \mathrm{C}$ and the nitrocellulose membrane was blocked for $1 \mathrm{~h}$ using Odyssey blocking buffer (Li-Cor; 927-40000), followed by overnight incubation with $1 / 500$ diluted anti-CLDN1 primary antibody at $4{ }^{\circ} \mathrm{C}$ (Invitrogen; 35-2500). Next, the membrane was incubated with $1 / 10000$ diluted anti-rabbit Dylight800 secondary antibody (Thermo Scientific; 35571) for $2 \mathrm{~h}$ on room temperature. To correct for loading differences, membranes were incubated with 1/10000 diluted primary anti-actin antibody (MP; 691002) overnight at $4{ }^{\circ} \mathrm{C}$, followed by incubation with anti-1/10000 diluted mouse Dylight680 secondary antibody (Thermo Scientific; 35518) for $1 \mathrm{~h}$ at room temperature. Next, the same blot was used to detect OCLN by incubation with $1 / 1000$ diluted primary antioccludin antibody (Life Technologies Europe B.V; 33-1500) overnight at $4{ }^{\circ} \mathrm{C}$, followed by for $2 \mathrm{~h}$ incubation on room temperature with $1 / 100000$ diluted secondary antibody (Thermo Scientific; 35521). Visualisation of the bands was done using Odyssey software and analysis was done in ImageStudio.

\subsection{Behavioural tests}

The cognitive behaviour of the mice was investigated using the basic protocol for the open field and novel object recognition test, as previously described (Antunes and Biala, 2012). Briefly, mice were brought into the testing room $30 \mathrm{~min}$ before the experiment to familiarize with the environment. To preclude the existence of olfactory cues, all objects were thoroughly cleaned with $20 \%$ ethanol after each trial. On the habituation day (day 0 ), mice were placed in a clear, plexiglass box $(40 \mathrm{~cm} \times 40 \mathrm{~cm} \times 40 \mathrm{~cm})$ and allowed to explore the arena without objects for $5 \mathrm{~min}$. Behavioural analysis included velocity $(\mathrm{cm} / \mathrm{s})$, total distance travelled $(\mathrm{m})$ and time spend in the center or borders of the arena. The next day, mice were subjected to the NOR test which includes 2 phases: the training and the testing phase. During the training trial, 2 identical objects were placed at 2 opposite positions within the box at the same distance from the nearest corner. Mice were allowed to explore the identical objects for $5 \mathrm{~min}$ and were then returned to their home cage. 15 min later, the mice were placed back in the same box, where 1 of the 2 familiar objects was switched to a novel one, and the $5 \mathrm{~min}$ testing phase was started. The time spent exploring each of the objects was defined as occurring when the 
mice touched the object with their mouth or nose. All trials were videotaped and scored manually. The percentage preference for the novel object was calculated using the following formula: percentage novel object preference $=$ (novel object exploration time $)$ (novel object exploration time + familiar object exploration tim e) ${ }^{*} 100 \%$. The NOR test is based on the spontaneous behaviour of mice to interact more with a novel object than with a familiar one. If the mouse explores the novel object for a longer time period, this indicates memory for the familiar object. If the mouse explores the novel and familiar objects for the same amount of time, this indicates a lack or loss of memory for the familiar object presented during the initial trial.

For the rotarod test (UB 47600), the drum of the rotarod (UB 47600) was slowly accelerated from a speed of 4-40 rpm over 300 $\mathrm{s}$. The latency to fall off the rotarod within this period was recorded. Mice received 5 consecutive trials without a training phase and the mean latency to fall off the rotarod was used for analysis.

\subsection{Statistics}

Data were analysed by Mann-Whitney test using GraphPad Prism or SPSS and are presented as means \pm standard error of mean (SEM), unless stated otherwise. Significance levels are indicated on the graphs ${ }^{*}, .01 \leq \mathrm{P}<.05$; ${ }^{* *}, .001 \leq \mathrm{P}<.01$; ${ }^{* * *}, .0001 \leq \mathrm{P}<.001$; $* * * *, \mathrm{P}<.0001$

\section{Results}

\subsection{Colonization by H. suis induces gastric inflammation}

One month after infection, colonization by $H$. suis was confirmed by analysing the stomach and small intestine. The mouse stomach consists of a non-glandular part and a glandular part. In this study, we analysed only the glandular part, consisting mainly of the corpus and the antrum of the stomach. As shown in Fig. 1a, the corpus and antrum of the stomach of $H$. suis-infected animals were colonized by an average of $4.51 \mathrm{E}+05$ and $7.30 \mathrm{E}+05$ bacteria per mg tissue, respectively. In contrast, no $H$. suis DNA was detected in the stomach of control mice (Fig. 1a). Additionally, $H$. suis DNA was also detected in the duodenum of infected mice but to a lower extent than in the stomach (Fig. 1a).

Analysis of H\&E-stained stomach of control animals showed no or negligible infiltration of mononuclear and polymorphonuclear cells in the mucosa and the submucosa, which agrees with the normal histological image of the stomach (Fig. 1b). In contrast, pronounced infiltration of mononuclear and polymorphonuclear cells was detected in the stomach of $H$. suis-infected animals (Fig. 1c), indicating gastric inflammation as scored by the Updated Sydney System (Fig. 1d). Furthermore, inflammation was also associated with changes in the expression levels of pro-inflammatory cytokines and chemokines. C57BL/6 mice have a typical Th17 response in the stomach when infected with $H$. suis (Flahou et al., 2012). Based on previous results in the research group, we therefore focused on some typical Th17 related genes: Il1 $\beta$, IL8 homologs (Lix and Kc), Il6, Il10, Il17 and Tnf. Gene expression analyses revealed that the mRNA levels of the murine interleukin 8 (IL8) homolog keratinocyte chemoattractant (Kc) and lipopolysaccharide-induced CXC chemokine (Lix) were significantly up-regulated in corpus (Fig. 1e) and in antrum (Fig. 1f) of $H$. suis-infected mice. A significant increase in gene expression of Il $1 \beta$ was observed only in the corpus of those animals (Fig. 1e) and a significant decrease of $I l 10$ in the antrum (Fig. 1f). Il6 and Tnf were not detected in stomach and duodenum. Additionally, a significant increase in protein levels of IL1 $\beta$ and IL17 was observed in the corpus of the stomach of $\mathrm{H}$. suis-infected mice (Fig. 1h-i). In contrast, no significant changes in the expression of cytokines and chemokines were found in the duodenum of mice infected with $H$. suis (Fig. 1g).

\subsection{Gastric H. suis infection induces inflammation and activation of microglia in the brain}

To determine whether $H$. suis affects the brain, cerebrospinal fluid (CSF) and brain tissue were analysed for inflammation. Bioplex analysis of CSF revealed no significant changes in cytokine and chemokine levels of IL1 $\beta$, KC, IL6 and IL10 (Fig. 2a), IL17 and TNF levels were not above the detection level. However, a significantly higher concentration of soluble tumour necrosis factor receptor 1 (sTNFR1) was found in the CSF of $H$. suis-infected mice (Fig. 2b). Despite the limited changes in the CSF, a significantly stronger expression of Il6 was detected in the brain hippocampus, while Il $1 \beta$, Tnf and $K c$ expression was unaffected (Fig. 2c). Lix, Il10 and $I l 17$ were not detected in the hippocampus. Since microglia are important mediators of brain inflammation, we stained brain sections for ionized calcium binding adaptor molecule 1 (IBA1), a marker for microglia (Fig. 2d-e). Automatic analysis showed a significant increase in the total IBA1-positive signal in the brain (Fig. 2f). Quantification of the number of microglia $/ \mathrm{mm}^{2}$ at the subventricular showed an increasing trend (Fig. $2 \mathrm{~g}$ ). These data indicate that $H$. suis activates a pro-inflammatory response in the brain. In parallel, we also analysed whether astrocytes were affected. Therefore, we performed an immunostaining to visualize astrocytes using anti-glial fibrillary acidic protein (GFAP), followed by confocal imaging (Fig. 2h-i) and automated analysis (Fig. 2j), but no significant difference could be observed.

Next, we determined whether $H$. suis infection influences cognition. Therefore, mice were subjected to the novel object recognition (NOR), open field and rotarod test. As shown in Fig. $2 \mathrm{k}, H$. suis infection results in loss of short-term memory in mice, revealing that the mild brain inflammation caused by $H$. suis infection negatively affects cognition. As shown in Fig. 2, H. suis-infected animals move with a higher velocity (Fig. 2l) and over a greater distance (Fig. 2m), compared to control mice. In contrast, no difference in the time spend in the center of the arena was observed (Fig. 2n). This suggests that $H$. suis infection doesn't affect anxiety, but does have an effect on activity. In agreement with the open field test, the rotarod test revealed that $H$. suis-infected mice are more active compared to control mice, reflected by a higher latency to fall (Fig. 2o).

\subsection{H. suis infection induces loss of the blood-CSF barrier but does not affect BBB integrity}

The presence of tight brain barriers, including the $\mathrm{BBB}$ and the blood-CSF barrier, ensure brain homeostasis (De Bock et al., 2014), but several inflammatory triggers are known to disrupt barrier integrity (Alvarez-Arellano and Maldonado-Bernal, 2014; Brkic et al., 2015; Gorle et al., 2016; Nielsen et al., 2012; Vandenbroucke et al., 2012). Here, we studied whether the effect of $H$. suis on the brain is associated with loss of barrier integrity. No change in BBB permeability was observed (Fig. 3a). In contrast to the BBB, a significant increase in blood-CSF barrier permeability was seen after one month of infection (Fig. 3b). Gene expression analysis of the blood-CSF barrier located at the choroid plexus revealed that several tight-junction genes were affected by $H$. suis infection. As shown in Fig. 3c, gene expression of claudin 1 (Cldn1) and occludin $(O \mathrm{cln})$ was significantly down-regulated in the presence of $H$. suis infection. Other tight-junction genes, such as Cldn5, zona occludens 1 (Zo1) and Zo3 decreased, but not significantly so. Next, immunostaining was performed to determine the subcellular localization of CLDN1 and ZO1. The arrowheads in Fig. 3d-g point to the loss of apical localization of CLDN1 and ZO1 in the choroid 
a

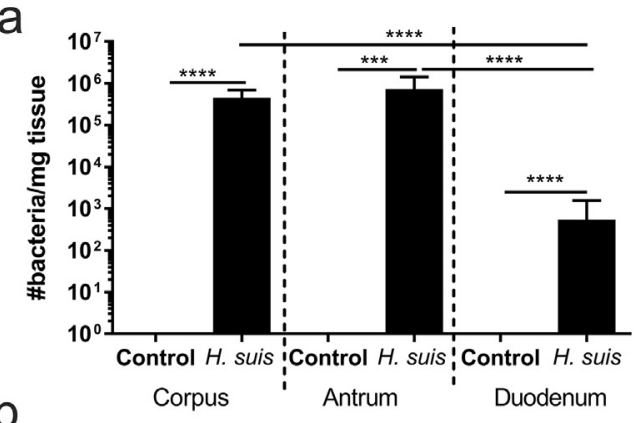

b

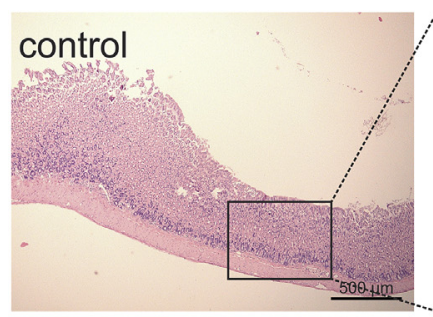

C

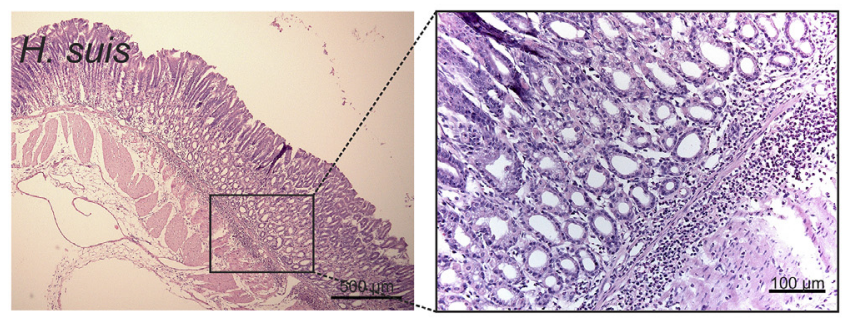

d

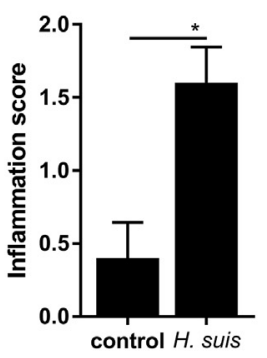

$\mathrm{h}$

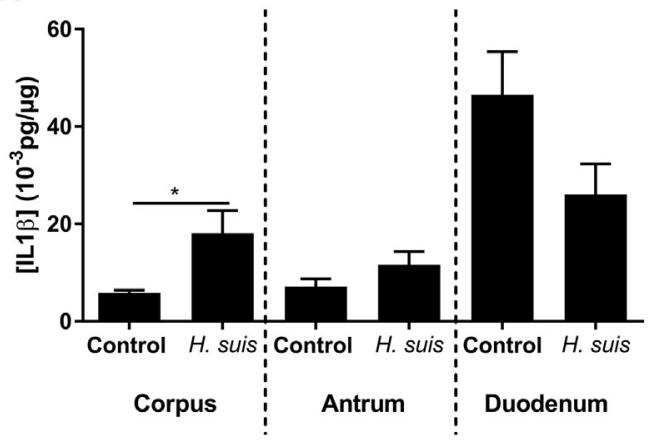

e

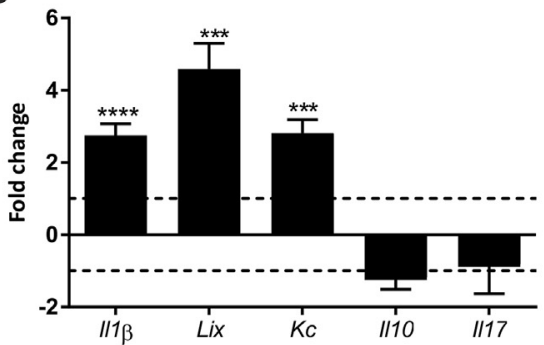

f

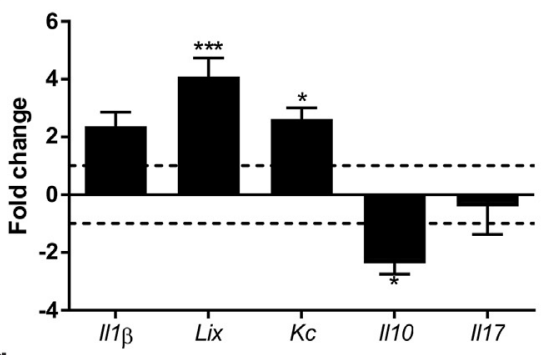

9
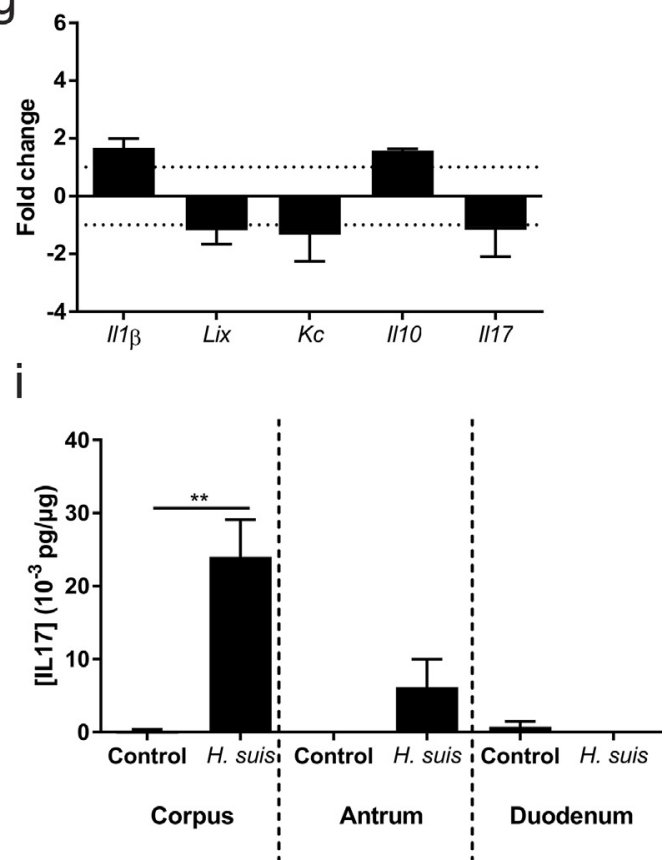

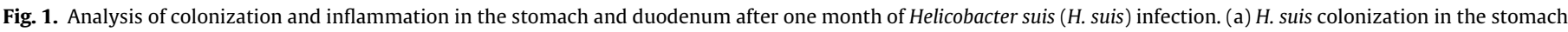

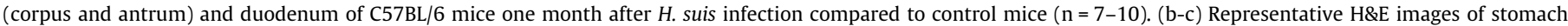

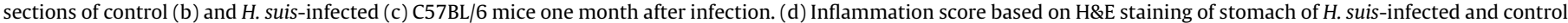

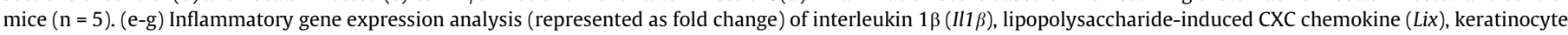

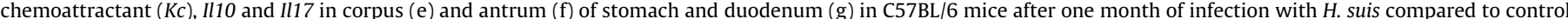

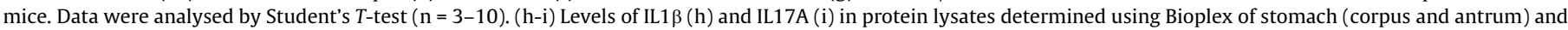
duodenum after inoculation with $H$. suis or growth medium. Data were analysed by Student's $t$-test $(n=5)$.

plexus epithelial cells of $H$. suis-infected mice. These data indicate that $H$. suis infection compromises the functionality of the bloodCSF barrier but does not affect the integrity of the BBB. Finally, western blot analysis was performed to determine the levels of CLDN1 and OCLN in the choroid plexus. As shown in Fig. 3h-k, the levels of both CLDN1 (Fig. 3h and j) and OCLN (Fig. 3i and k) are significantly lower after 1 month of $H$. suis infection.

\subsection{H. suis infection induces loss of gastric mucosal barrier integrity and subsequent systemic inflammation}

Increased gastrointestinal permeability, which could lead to leakage of pro-inflammatory mediators and bacterial metabolites from the gut into the systemic circulation, is considered one of the gut-brain axis pathways (Cryan and Dinan, 2012; Mayer et al., 2014). In our study, we used oral gavage of FITC-dextran to investigate the influence of gastric $H$. suis infection on the gastrointestinal barrier. Plasma fluorescence analysis revealed a significant increase in gastrointestinal permeability after one month of $\mathrm{H}$. suis infection (Fig. 4a). The gastric mucosal barrier is composed of the gastric mucosa (epithelium, lamina propria and muscularis mucosa), with a mucus layer on top (Caron et al., 2015; Kawakubo et al., 2004; Kufe, 2009; Linden et al., 2002; McGuckin et al., 2011; Yamashiro, 2006). This gastric mucus layer, consisting mainly of secreted mucins, is the first barrier pathogens encounter. The membrane-associated mucins located underneath this mucus 
a

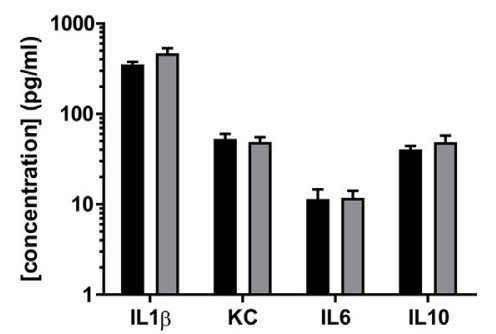

d

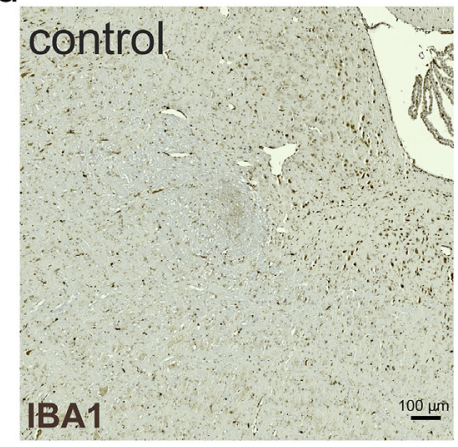

$\mathrm{h}$

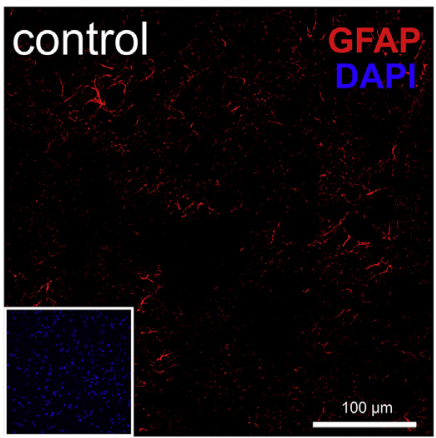

$\mathrm{k}$

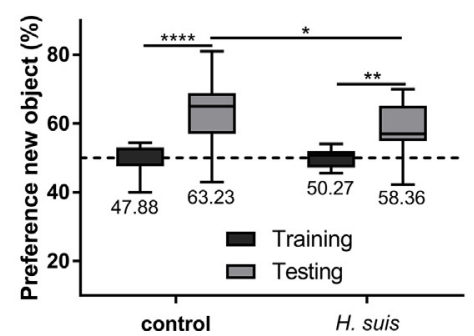

b

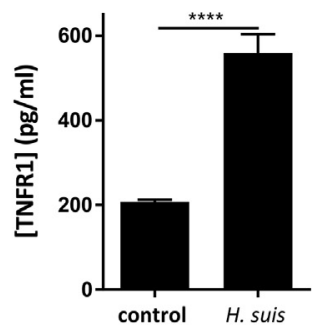

e

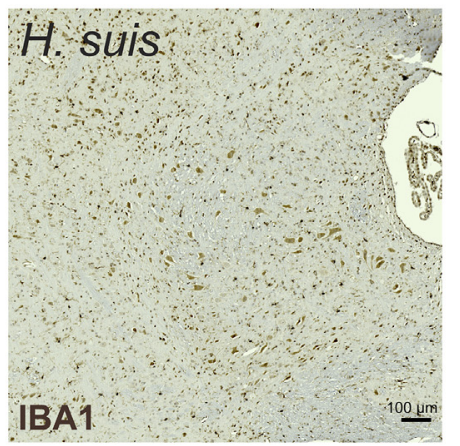

i

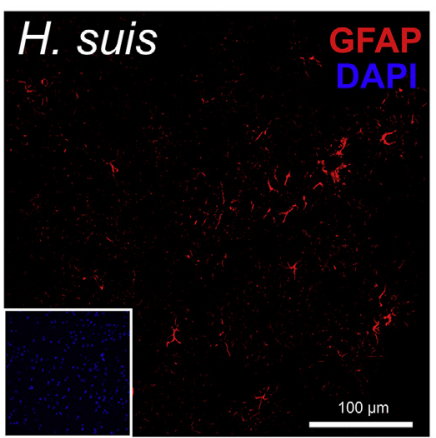

C

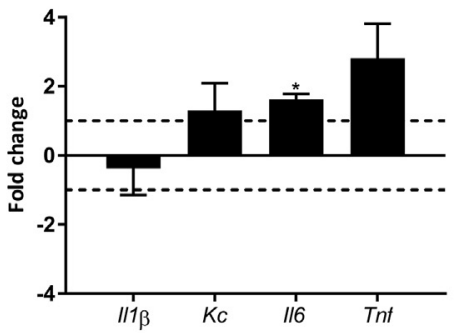

f

9
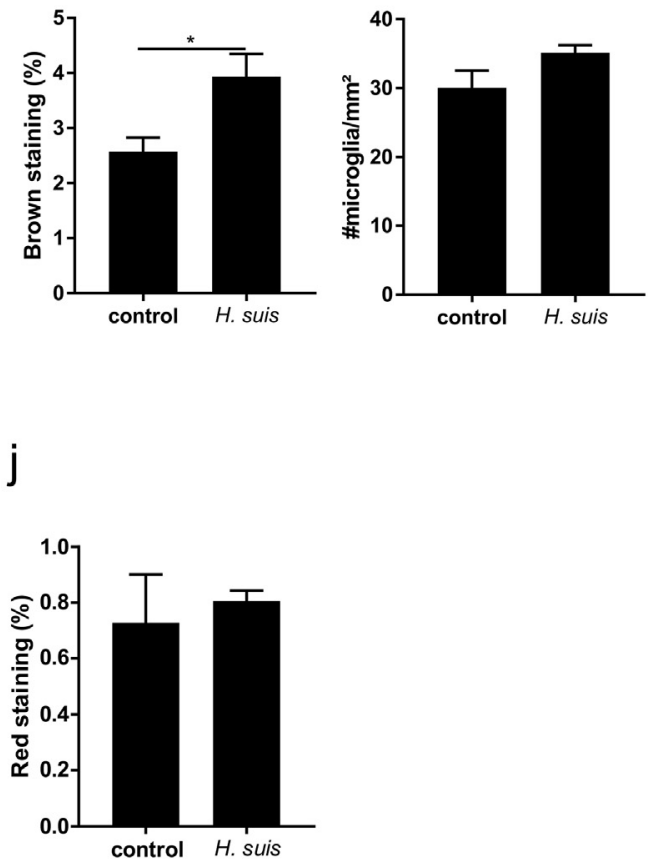

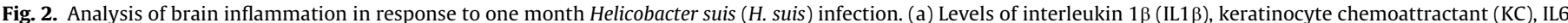

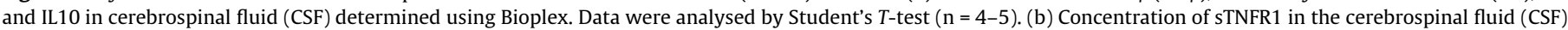

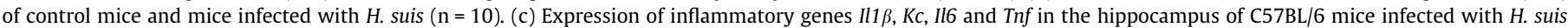

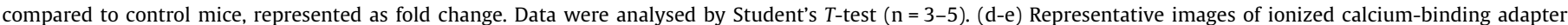

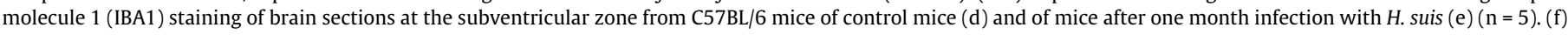

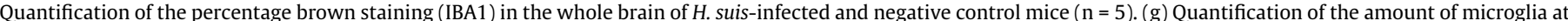

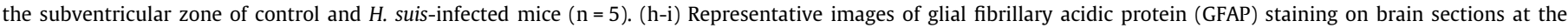

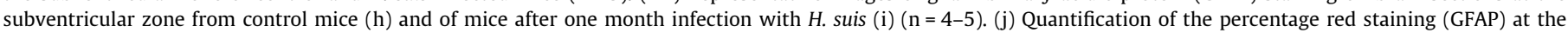

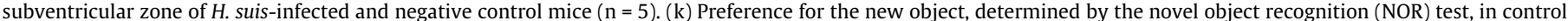

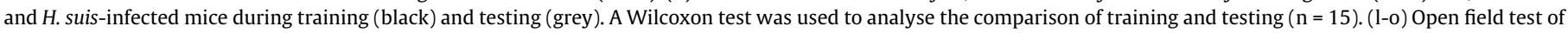

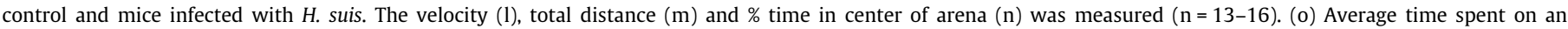
accelerating rotarod of control and mice infected with $H$. suis $(\mathrm{n}=14-15)$.

layer also provide a barrier, thereby limiting access to the epithelial cell surface (McGuckin et al., 2011). Changes in mucin distribution and expression might affect the integrity of the gastric mucosal barrier (Kufe, 2009). Therefore, we analysed gastric mucin expression. RT-qPCR analysis revealed a significantly increased gene expression of the transmembrane mucin 13 (Muc13) in the corpus 
a

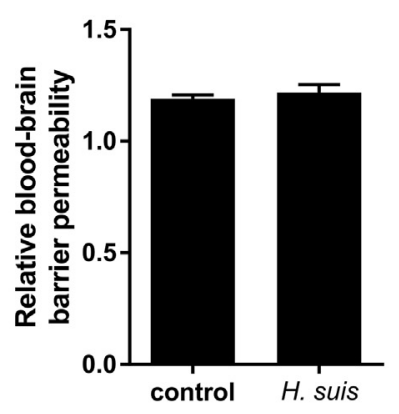

b

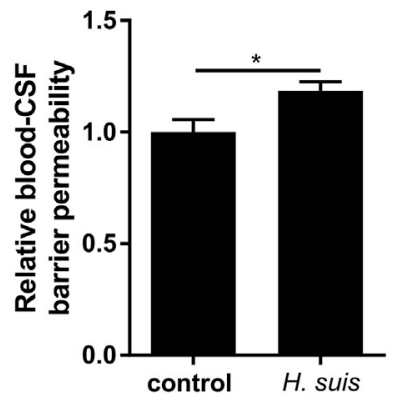

C

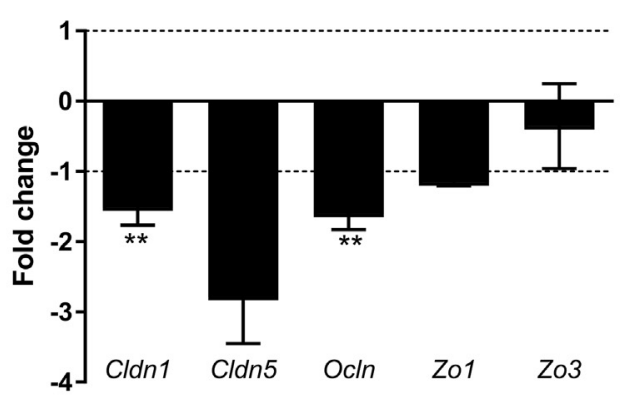

d

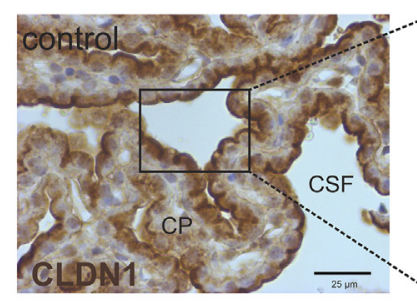

f

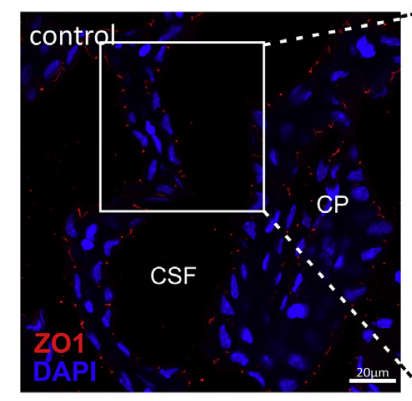

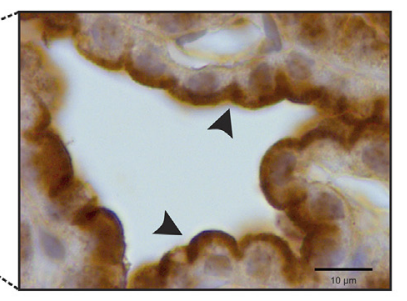

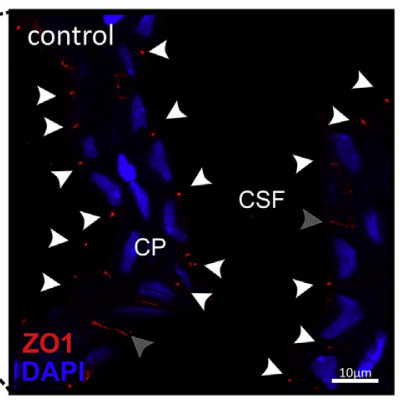

h

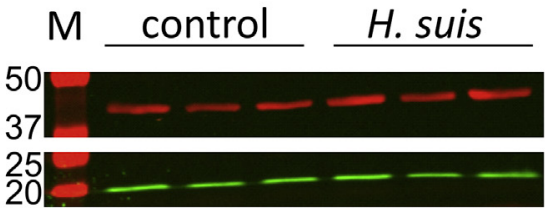

actin CLDN1
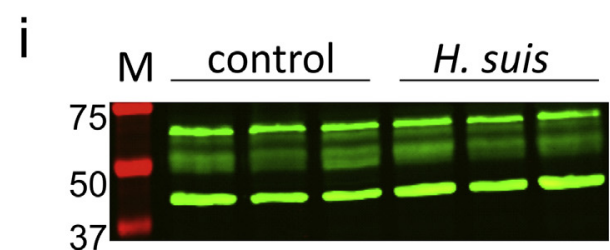
actin e
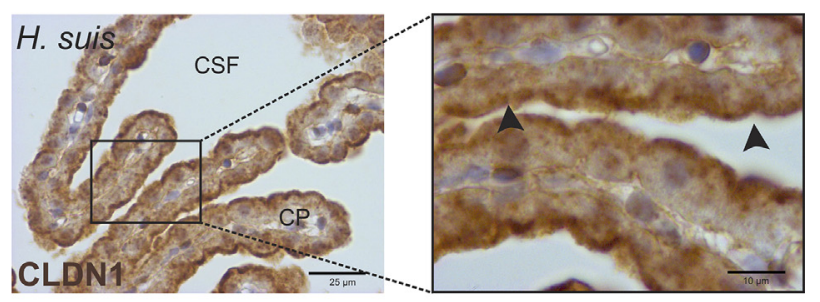

g
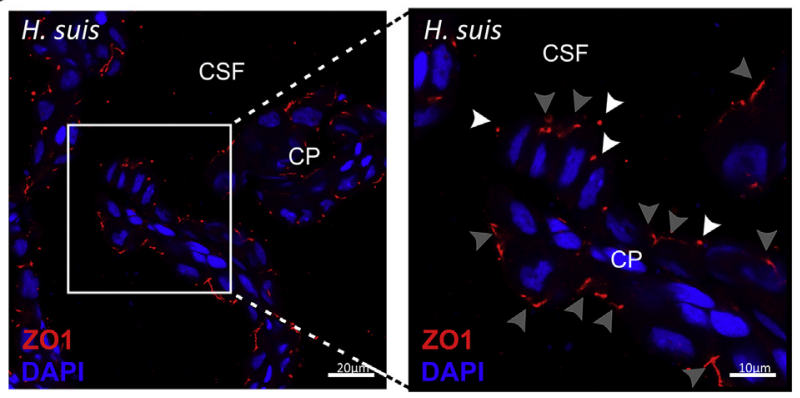

k

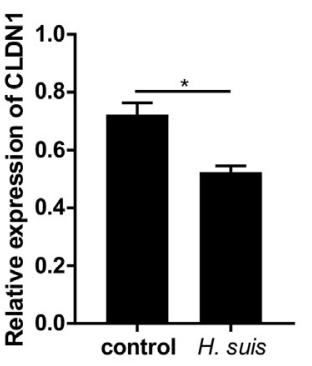

.

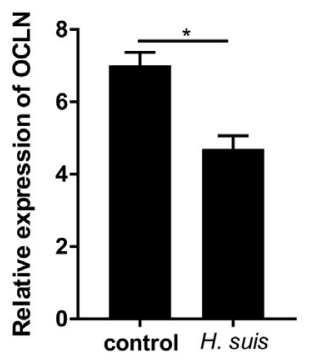

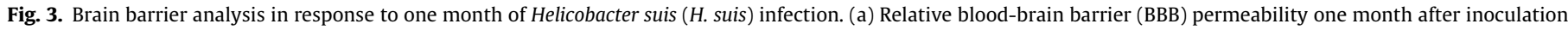

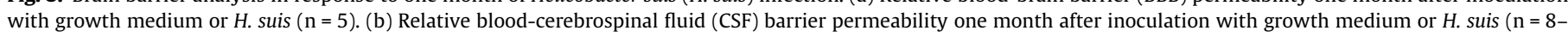

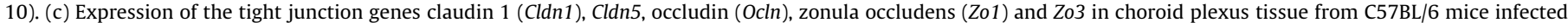

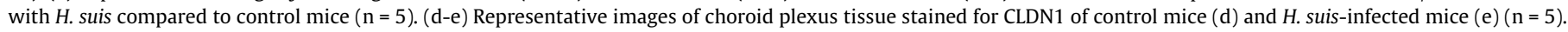

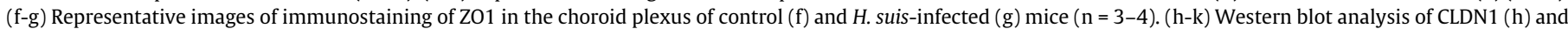

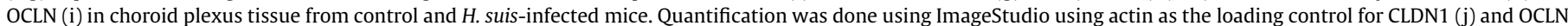
(k) $(\mathrm{n}=3)$. (Abbreviations: CLDN1, claudin-1; CP, choroid plexus; CSF, cerebrospinal fluid; OCLN, occludin).

of $H$. suis-infected mice, while the expression of Muc5ac, Muc5b and Muc6 were unaffected (Fig. 4b). This finding was confirmed histologically (Fig. 4e-f): compared to control mice (Fig. 4e), apical membrane and cytoplasmatic MUC13 staining (brown) was much more pronounced in the mucus-secreting epithelial cells of the stomach of $H$. suis-infected mice (Fig. 4f). In contrast, Muc13 gene expression did not change in antrum and was significantly down-regulated in the duodenum of $H$. suis-infected animals (Fig. 4c-d).

The gastric epithelium located underneath the mucus layer is formed by epithelial cells connected by tight junction proteins (Caron et al., 2015). We performed immunostaining to determine 
a

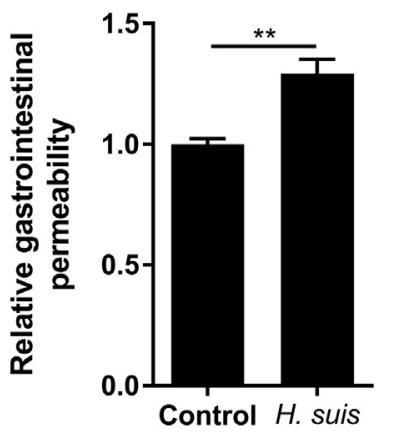

b

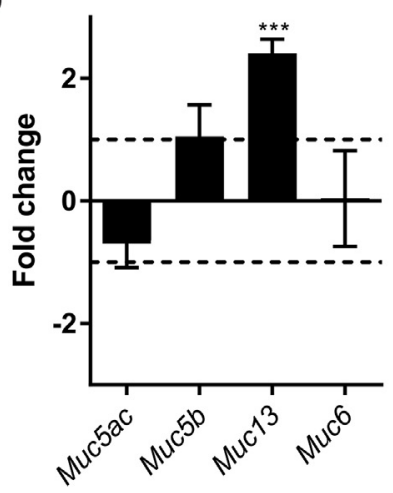

C

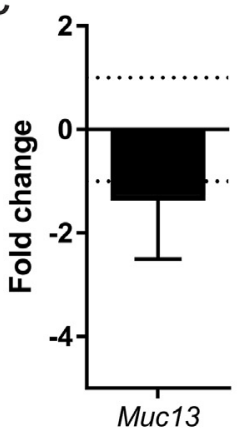

d

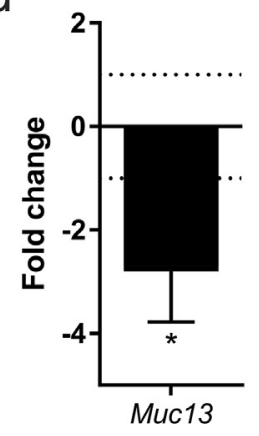

e

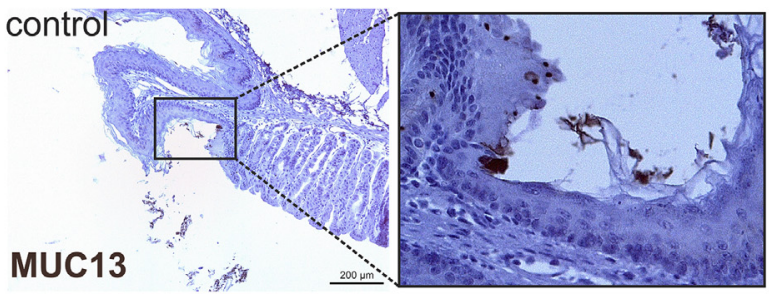

$f$

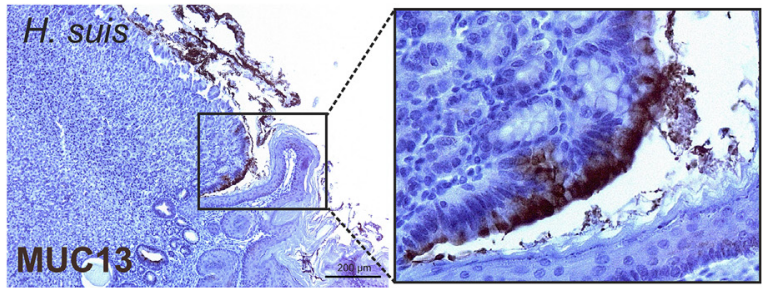

$\mathrm{h}$
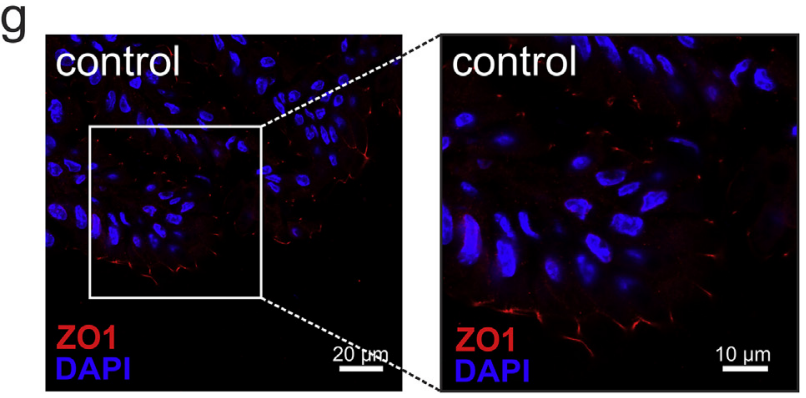

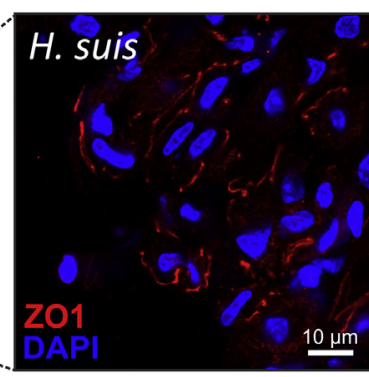

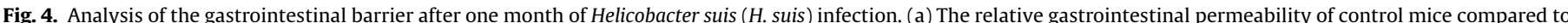

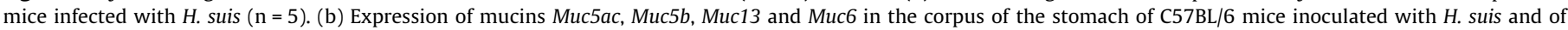

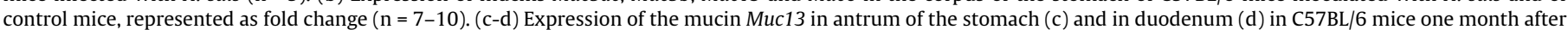

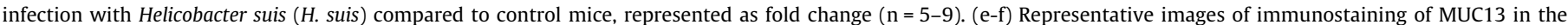

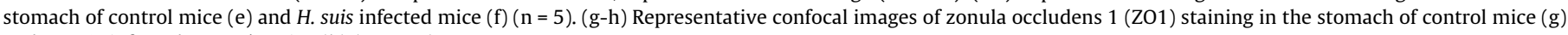
and $H$. suis-infected C57BL/6 mice $(\mathrm{h})(\mathrm{n}=4-5)$.

whether the $H$. suis-induced alterations in gastrointestinal barrier integrity were correlated with changes in junctional proteins in the stomach. Confocal imaging revealed that expression of ZO1 was disturbed. Compared to control mice, in which ZO1 was located mainly at the apical side of the stomach epithelial cells (Fig. 4g), the ZO1 signal was much more diffuse in stomach samples from $\mathrm{H}$. suisinfected animals (Fig. 4h). The changes in expression and localisation of both MUC13 and ZO1 might thus explain the increased gastrointestinal leakage in the presence of $H$. suis infection.

Disruption of the gastric mucosal barrier might result in leakage of $H$. suis into the periphery and subsequent translocation into the brain. However, neither RT-qPCR analysis on hippocampal tissue and anti-Helicobacter immunostaining on brain sections could show H. suis in the brain as displayed in Fig. 5a-f.

Alternatively, also pro-inflammatory cytokines, chemokines and bacterial metabolites or fragments might leak from the stomach into the blood, leading to systemic inflammation (Clapp et al., 2017; Cryan and Dinan, 2012; Fung et al., 2017;
Kelly et al., 2017; Tognini, 2017). Additionally, recent studies indicated that alterations in circulating cytokine levels may directly influence the functionality of the brain barriers and the brain (Balusu et al., 2016b; Cryan and Dinan, 2012; Stridh et al., 2013; Vandenbroucke et al., 2012; Varatharaj and Galea, 2016).

Therefore, the presence of systemic inflammation was evaluated by analysing serum of control and $H$. suis-infected mice. As shown in Fig. 5g, $H$. suis infection leads to a significant increase in Toll-Like Receptor 4 (TLR4) ligands in the serum. Additionally, as shown in Fig. 5h, the level of the pro-inflammatory cytokine IL1 $\beta$ increased significantly in the serum of $H$. suis-infected mice, while IL6 and TNF serum levels were below detection limit (data not shown). These findings indicate that the loss of blood-CSF barrier integrity observed in our study might have been caused by a low grade, systemic inflammation induced by $H$. suis. In agreement with this finding, gene expression analysis of choroid plexus tissue revealed a significantly increased expression of pro-inflammatory cytokine Il1 $\beta$ (Fig. 5i). 
a
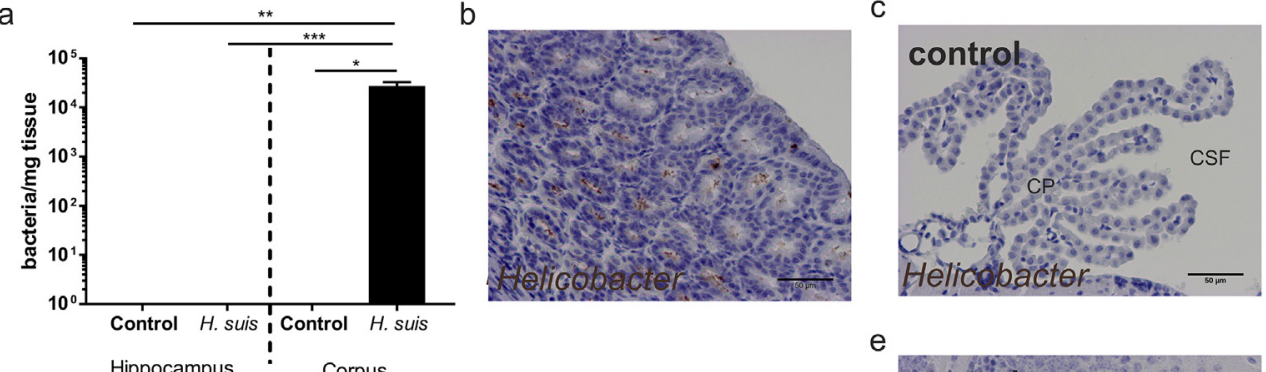

e

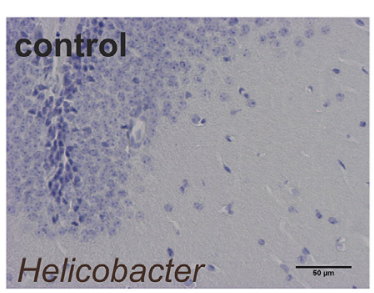

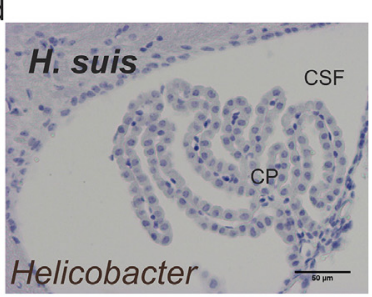

f

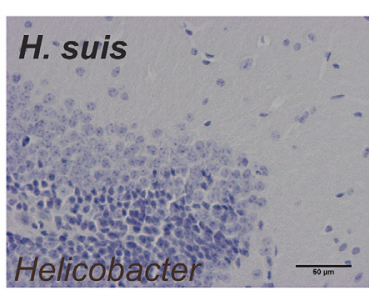

g

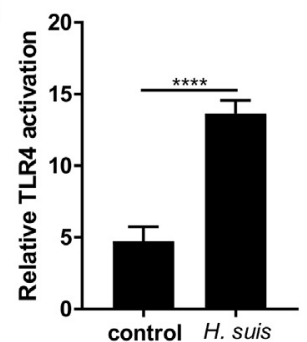

$\mathrm{h}$

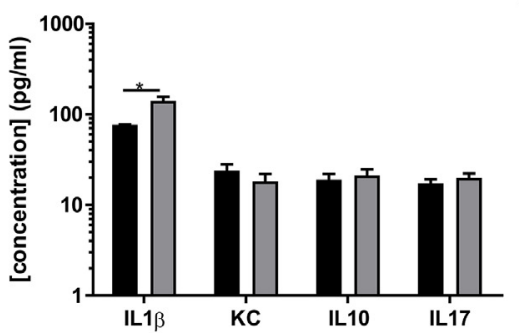

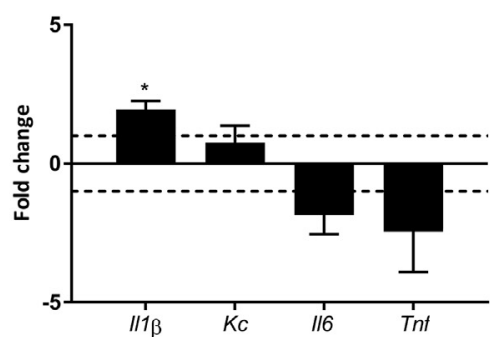

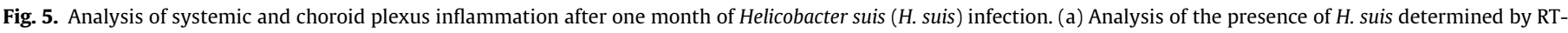

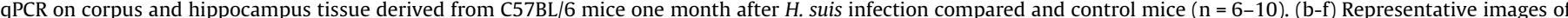

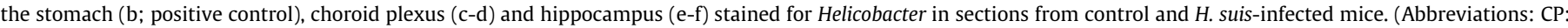

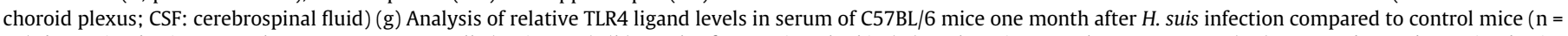

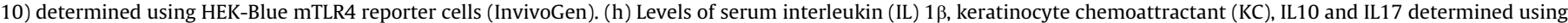

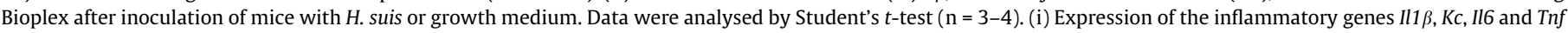
in choroid plexus tissue of C57BL/6 mice inoculated with $H$. suis or growth medium, represented as fold change $(\mathrm{n}=3-5)$.

\section{Discussion}

Alterations in the gut microbiome are believed to contribute to many different human health problems (Bercik et al., 2011; Boulange et al., 2016; Bravo et al., 2011; Clapp et al., 2017; Clemente et al., 2012; Dinan and Cryan, 2017b; Fung et al., 2017; Kelly et al., 2017; Ochoa-Reparaz and Kasper, 2014; Parracho et al., 2005; Tognini, 2017; Walters et al., 2014; Zhang et al., 2015). Next to the direct effects H. pylori infection on the gastrointestinal system (Camilo et al., 2017; Johnson and Ottemann, 2017; Kusters et al., 2006; Li et al., 2016; Mayer et al., 2014; PereVedrenne et al., 2017; Teng et al., 2017), it has been associated with the occurrence of different neurodegenerative disorders, including Alzheimer's disease (Alvarez-Arellano and MaldonadoBernal, 2014; Doulberis et al., 2017; Roubaud Baudron et al., 2016; Wirdefeldt et al., 2011; Wong et al., 2014) and Parkinson's disease (Alvarez-Arellano and Maldonado-Bernal, 2014; Blaecher et al., 2013; Dinan and Cryan, 2017a; Mridula et al., 2017; Mulak and Bonaz, 2015; Wirdefeldt et al., 2011; Wong et al., 2014). We recently highlighted a similar association of gastric $H$. suis infections in Parkinson's disease (Blaecher et al., 2013). However, it remains unknown how gastric Helicobacter infection in general influences the brain.

The gastrointestinal system and the brain are connected through neural, endocrine and immune pathways (Dinan and Cryan, 2017b). Here, we observed that one month of infection with $H$. suis resulted in increased brain inflammation. In particular, we observed a moderate but significant up-regulation of Il6 gene expression in the hippocampus, elevated levels of sTNFR1 in CSF (a biomarker for Alzheimer's disease (Jiang et al., 2011)), and more microglial IBA1 positivity in the brain. Interestingly, elevated brain levels of IL6 have also been reported in traumatic brain injury (Goodman et al., 1990; Kossmann et al., 1995), depression (Brietzke et al., 2009), Parkinson's disease (Boka et al., 1994) and Alzheimer's disease (Angelopoulos et al., 2008), suggesting a role for IL6 in the above-mentioned neuropathologies. Furthermore, microglia activation has been observed in $H$. pylori infection (Lo et al., 2009), and priming of microglia by prior pathology has shown to accelerate brain pathology (Cunningham, 2013). Strikingly, we observed that the limited $H$. suis-induced increase in brain inflammation is associated with a decrease in cognitive decline, as determined via the NOR test. Additionally, higher activity was observed in both the open field and rotarod test, which has been linked to Parkinson's disease before, namely in the A53T model of Parkinson's disease (Paumier et al., 2013). While no data are available on $H$. suis, it has been shown that the cognitive state of $H$. pylori positive mild cognitive impairment $(\mathrm{MCI})$ patients correlated with serum anti-H. pylori IgG concentration, suggesting that infection might partially contribute to the pathophysiology of MCI (Kountouras et al., 2007).

As mentioned, neural, endocrine and immune pathways connect the gut and the brain. However, a series of barriers, including the BBB and the blood-CSF barrier (De Bock et al., 2014) prevent direct contact between blood and brain and assure brain homeostasis. Our results indicate that gastric $H$. suis infection induces leakage of the blood-CSF barrier but not of the BBB. One month after $H$. suis infection, we observed increased leakiness of the blood-CSF barrier, which will subsequently affect CSF and brain 
parenchyma upon CSF-interstitial fluid (ISF) exchange. This lowgrade brain inflammation might be below the threshold to induce loss of BBB integrity. Additionally, we can't exclude that the method used is not sensitive enough to detect BBB leakage. The blood-CSF barrier has a lower transepithelial electrical resistance compared to the BBB (Gorle et al., 2016), making it more sensitive to inflammatory triggers (both from the periphery and the brain). Of note, the blood-CSF barrier is also more dynamic and is seen as an immune sensor for the central nervous system (Shechter et al., 2013; Strazielle and Ghersi-Egea, 2000). The disturbance of the blood-CSF barrier was accompanied by loss of tight junctions, and specifically by a significant decrease in expression of Cldn1, Ocln and disturbed localization of CLDN1 and ZO1 protein in the choroid plexus epithelium. The choroid plexus epithelium is uniquely positioned between blood and brain, and we and others believe that this blood-CSF interface is an essential player in the communication between the periphery and the brain. Indeed, several studies reported that choroid plexus epithelial cells sense inflammatory changes in the periphery, which in turn induces changes in functionality of the choroid plexus epithelial cells (Balusu et al., 2016b; Marques et al., 2009, 2007; Thouvenot et al., 2006; Vandenbroucke et al., 2012). The response of the choroid plexus can vary depending on the nature and duration of the stimuli. A single, acute injection of LPS, leads to rapid changes in the transcriptome of the choroid plexus, with a large number of genes which are massively upregulated. A more attenuated response is seen when the stimulus is repeated (Marques and Sousa, 2015). This is also observed in models of multiple sclerosis and patients with major depressive disorders. Expression of proinflammatory mediators, such as LPS, IL1, TNF $\alpha$ and IFN $\gamma$, can cause breakdown of the blood-CSF barrier, but can also induce the expression of trafficking molecules in the choroid plexus (Kunis et al., 2013; Schwerk et al., 2010; Steffen et al., 1996; Zeni et al., 2007). This leads to infiltration of T cells and macrophages, which supports central nervous system repair (Kunis et al., 2013). In a model of spinal cord injury, the choroid plexus is important in the recovery, by recruiting anti-inflammatory M2 macrophages and CD4+ $\mathrm{T}$ cells to the injury (Shechter et al., 2013). However, immune trafficking is not always beneficial. For example, viral infection can spread to the brain via the upregulation of immune cell trafficking at the choroid plexus (Meeker et al., 2012).

In agreement with these studies, we observed $H$. suis-induced systemic inflammation, characterized by increased levels of TLR4 ligands and IL1 $\beta$ in the serum, and this resulted in increased inflammation at the choroid plexus, loss of blood-CSF barrier integrity and subsequently brain inflammation.

The observed $H$. suis-induced changes in the choroid plexus are small but significant, which is in line with the literature. Indeed, it has been shown that the response of the choroid plexus to an acute peripheral stimulation is rapid, strong and transient, while its response is weaker, but persistent in case of a continuous stimulation (Marques and Sousa, 2015). H. suis-induced blood-CSF barrier leakage and associated dysfunction of the choroid plexus epithelium is known to be destructive to the brain (Balusu et al., 2016a; Johanson et al., 2011; Marques et al., 2007; Redzic et al., 2005). In agreement with this, increased blood-CSF barrier permeability has been observed in several neuroinflammatory disorders, including Alzheimer's disease, aging and Parkinson's disease (Balusu et al., 2016a; Brkic et al., 2015; Gorle et al., 2016; Marques et al., 2013; Pisani et al., 2012). Consequently, this suggests that the $H$. suis-induced increased blood-CSF barrier leakage contributes to the observed neuroinflammation and cognitive decline and increased activity. Both the higher activity in the open field test and the higher latency to fall in the rotarod test have been linked to Parkinson's disease before, namely in the A53T model of Parkinson's disease (Paumier et al., 2013).
Disruption of the gastric mucosal barrier results in leakage of inflammatory cytokines, chemokines and/or bacterial components from the stomach into the systemic circulation (Cryan and Dinan, 2012; Mayer et al., 2014), thereby inducing systemic inflammation. In our study, the extent of colonization of the stomach by H. suis was similar to what has been reported (Flahou et al., 2010). DNA from $H$. suis was also found in the duodenum, but it is not clear whether this pathogen colonized the duodenum or, otherwise, the qRT-PCR picked up DNA from bacteria colonizing the stomach. $H$. suis infection evoked a pro-inflammatory immune response in the stomach characterized by infiltration of innate immune cells and up-regulation of IL1 $\beta$ and murine IL8 homologue genes Lix and $K c$; these observations are in line with previous studies (Bosschem et al., 2017a, b; Flahou et al., 2012; Flahou et al., 2010). Inflammation in the stomach was also accompanied by increased gastrointestinal barrier permeability and changes in the localization of the tight junction protein ZO1. Concordant with our study, co-culture of primary human gastric epithelial cells with H. pylori resulted in the disruption of membrane ZO1 (Wroblewski and Peek, 2011).

Aberrant expression of transmembrane mucins has also been shown to play a role in disturbance of mucosal barrier integrity (Kufe, 2009). In our study, an inappropriate overexpression of the membrane-associated mucin MUC13 was seen. This mucin, which is predominantly expressed in the intestines and only at a very low level in the stomach, seems to be induced by IL1 $\beta$ in the inflamed stomach during Helicobacter infection (Cheng et al., 2016). Inappropriate overexpression of transmembrane mucins can affect gastric mucosal barrier integrity by disrupting cell polarity and cell-cell interactions (Kufe, 2009), but further investigations are necessary to confirm the role of MUC13 in gastric mucosal barrier dysfunction. Taken together, gastric $H$. suis infection-induced inflammation in the stomach, which was associated with gastric mucosal damage resulting in leakage of inflammatory mediators into the blood and leading to systemic inflammation.

In conclusion, we identified a pivotal role of the choroid plexus in the communication between the gastrointestinal tract and the brain. Our results indicate that $H$. suis induces alterations in the brain, including inflammation and cognitive decline, via loss of blood-CSF barrier integrity in response to $H$. suis-induced leakage of the gastrointestinal barrier and subsequent systemic inflammation.

\section{Acknowledgments}

Declaration of funding interests: This work was supported by the Research Fund of Ghent University, Belgium (1G01014 and 01IO0714) and by the Research Foundation Flanders (FWO Vlaanderen). The authors thank Sofie De Bruyckere for her excellent technical assistance and the VIB Bio Imaging Core for suggestions concerning immunohistochemistry and imaging.

\section{Authors contribution}

R.E.V., F.H. and A.S. were joint principal investigators. N.G., C.B. and E.B. contributed equally. N.G., C.B., E.V., S.B., J.V., C.V., C.V.C., E. V.W., G.V.I., R.E.V. and C.L. performed the laboratory work. N.G., C. B., A.S. and R.E.V. collected and analysed the data. R.D., Cl.L. and F. H. advised on parts of the study. N.G., C.B., A.S. and R.E.V. wrote the paper. N.G., C.B., R.D., Cl.L., F.H., A.S. and R.E.V. reviewed the manuscript.

\section{Conflict of Interest}

The authors declare no competing financial interests. 


\section{References}

Alvarez-Arellano, L., Maldonado-Bernal, C., 2014. Helicobacter pylori and neurological diseases: married by the laws of inflammation. World J. Gastrointest. Pathophysiol. 5, 400-404.

Angelopoulos, P., Agouridaki, H., Vaiopoulos, H., Siskou, E., Doutsou, K., Costa, V., Baloyiannis, S.I., 2008. Cytokines in Alzheimer's disease and vascular dementia. Int. J. Neurosci. 118, 1659-1672.

Antunes, M., Biala, G., 2012. The novel object recognition memory: neurobiology, test procedure, and its modifications. Cogn. Process. 13, 93-110.

Baele, M., Decostere, A., Vandamme, P., Ceelen, L., Hellemans, A., Mast, J., Chiers, K., Ducatelle, R., Haesebrouck, F., 2008. Isolation and characterization of Helicobacter suis sp. nov. from pig stomachs. Int. J. Syst. Evol. Microbiol. 58, 1350-1358.

Balusu, S., Brkic, M., Libert, C., Vandenbroucke, R.E., 2016a. The choroid plexuscerebrospinal fluid interface in Alzheimer's disease: more than just a barrier. Neural Regen. Res. 11 (4), 534-537 [PMID:27212900].

Balusu, S., Van Wonterghem, E., De Rycke, R., Raemdonck, K., Stremersch, S., Gevaert, K., Brkic, M., Demeestere, D., Vanhooren, V., Hendrix, A., Libert, C. Vandenbroucke, R.E., 2016b. Identification of a novel mechanism of blood-brain communication during peripheral inflammation via choroid plexus-derived extracellular vesicles. EMBO Mol. Med., 1162-1183.

Bercik, P., Denou, E., Collins, J., Jackson, W., Lu, J., Jury, J., Deng, Y., Blennerhassett, P., Macri, J., McCoy, K.D., Verdu, E.F., Collins, S.M., 2011. The intestinal microbiota affect central levels of brain-derived neurotropic factor and behavior in mice. Gastroenterology 141, 599-609. 609 e591-593.

Bergen, A.A., Kaing, S., ten Brink, J.B., Netherlands Brain, B., Gorgels, T.G., Janssen, S. F., 2015. Gene expression and functional annotation of human choroid plexus epithelium failure in Alzheimer's disease. BMC Genomics 16, 956.

Blaecher, C., Smet, A., Flahou, B., Pasmans, F., Ducatelle, R., Taylor, D., Weller, C., Bjarnason, I., Charlett, A., Lawson, A.J., Dobbs, R.J., Dobbs, S.M., Haesebrouck, F., 2013. Significantly higher frequency of Helicobacter suis in patients with idiopathic parkinsonism than in control patients. Aliment. Pharmacol. Ther. 38, 1347-1353.

Boka, G., Anglade, P., Wallach, D., Javoy-Agid, F., Agid, Y., Hirsch, E.C., 1994. Immunocytochemical analysis of tumor necrosis factor and its receptors in Parkinson's disease. Neurosci. Lett. 172, 151-154.

Bosschem, I., Flahou, B., Bakker, J., Heuvelman, E., Langermans, J.A, De Bruyne, E., Joosten, M., Smet, A., Ducatelle, R., Haesebrouck, F., 2017a. Comparative virulence of in vitro-cultured primate- and pig-associated Helicobacter suis strains in a BALB/c mouse and Mongolian gerbil model. Helicobacter 22 (2) [PMID:27558281]

Bosschem, I., Flahou, B., Van Deun, K., De Koker, S., Volf, J., Smet, A., Ducatelle, R., Devriendt, B., Haesebrouck, F., 2017b. Species-specific immunity to Helicobacter suis. Helicobacter, 22.

Boulange, C.L., Neves, A.L., Chilloux, J., Nicholson, J.K., Dumas, M.E., 2016. Impact of the gut microbiota on inflammation, obesity, and metabolic disease. Genome Med. 8, 42.

Bravo, J.A., Forsythe, P., Chew, M.V., Escaravage, E., Savignac, H.M., Dinan, T.G., Bienenstock, J., Cryan, J.F., 2011. Ingestion of Lactobacillus strain regulates emotional behavior and central GABA receptor expression in a mouse via the vagus nerve. Proc. Natl. Acad. Sci. U.S.A. 108, 16050-16055.

Brietzke, E., Stertz, L., Fernandes, B.S., Kauer-Sant'anna, M., Mascarenhas, M., Escosteguy Vargas, A., Chies, J.A., Kapczinski, F., 2009. Comparison of cytokine levels in depressed, manic and euthymic patients with bipolar disorder. J. Affect. Disord. 116, 214-217.

Brkic, M., Balusu, S., Van Wonterghem, E., Gorle, N., Benilova, I., Kremer, A., Van Hove, I., Moons, L., De Strooper, B., Kanazir, S., Libert, C., Vandenbroucke, R.E., 2015. Amyloid beta oligomers disrupt blood-CSF barrier integrity by activating matrix metalloproteinases. J. Neurosci. 35, 12766-12778.

Camilo, V., Sugiyama, T., Touati, E., 2017. Pathogenesis of Helicobacter pylori infection. Helicobacter 22 (Suppl.), 1.

Caron, T.J., Scott, K.E., Fox, J.G., Hagen, S.J., 2015. Tight junction disruption: Helicobacter pylori and dysregulation of the gastric mucosal barrier. World J. Gastroenterol. 21, 11411-11427.

Cheng, L., Mirko, R., Sara, L., Medea, P., Caroline, B., Eva, B., Myrthe, J., Bram, F., Wim, V.D., Richard, D., Freddy, H., Annemieke, S., 2016. The Helicobacter heilmannii hofE and hoff genes are essential for colonization of the gastric mucosa and play a role in IL-1beta-induced gastric MUC13 expression. Helicobacter 21, 504-522.

Clapp, M., Aurora, N., Herrera, L., Bhatia, M., Wilen, E., Wakefield, S., 2017. Gut microbiota's effect on mental health: the gut-brain axis. Clin. Pract. 7, 987.

Clemente, J.C., Ursell, L.K., Parfrey, L.W., Knight, R., 2012. The impact of the gut microbiota on human health: an integrative view. Cell 148, 1258-1270.

Cryan, J.F., Dinan, T.G., 2012. Mind-altering microorganisms: the impact of the gut microbiota on brain and behaviour. Nat. Rev. Neurosci. 13, 701-712.

Cunningham, C., 2013. Microglia and neurodegeneration: the role of systemic inflammation. Glia 61, 71-90.

De Bock, M., Vandenbroucke, R.E., Decrock, E., Culot, M., Cecchelli, R., Leybaert, L., 2014. A new angle on blood-CNS interfaces: a role for connexins? FEBS Lett. $588,1259-1270$.

De Cooman, L., Flahou, B., Houf, K., Smet, A., Ducatelle, R., Pasmans, F., Haesebrouck, F., 2013. Survival of Helicobacter suis bacteria in retail pig meat. Int. J. Food Microbiol. 166, 164-167.
Demeestere, D., Libert, C., Vandenbroucke, R.E., 2015. Clinical implications of leukocyte infiltration at the choroid plexus in (neuro)inflammatory disorders. Drug Discov. Today 20, 928-941.

Dinan, T.G., Cryan, J.F., 2017a. Gut-brain axis in 2016: brain-gut-microbiota axis mood, metabolism and behaviour. Nat. Rev. Gastroenterol. Hepatol. 14, 69-70.

Dinan, T.G., Cryan, J.F., 2017b. The microbiome-gut-brain axis in health and disease Gastroenterol. Clin. North Am. 46, 77-89.

Dobbs, S.M., Dobbs, R.J., Smet, A., Weller, C., Charlett, A., Bjarnason, I., Lawson, A.J., Ibrahim, M.A.A., Taylor, D., Blaecher, C., Pasmans, F., Ducatelle, R., Flahou, B., Haesebrouck, F., 2013. Clinical significance of Helicobacter suis in idiopathic Parkinsonism. Helicobacter 18. 149-149.

Doulberis, M., Kotronis, G., Thomann, R., Polyzos, S.A., Boziki, M., Gialamprinou, D., Deretzi, G., Katsinelos, P., Kountouras, J., 2017. Impact of Helicobacter pylori on Alzheimer's disease: what do we know so far? Helicobacter.

Flahou, B., Deun, K.V., Pasmans, F., Smet, A., Volf, J., Rychlik, I., Ducatelle, R. Haesebrouck, F., 2012. The local immune response of mice after Helicobacter suis infection: strain differences and distinction with Helicobacter pylori. Vet. Res. 43, 75.

Flahou, B., Haesebrouck, F., Pasmans, F., D'Herde, K., Driessen, A., Van Deun, K., Smet, A., Duchateau, L., Chiers, K., Ducatelle, R., 2010. Helicobacter suis causes severe gastric pathology in mouse and mongolian gerbil models of human gastric disease. PLoS One 5, e14083.

Foster, J.A., McVey Neufeld, K.A., 2013. Gut-brain axis: how the microbiome influences anxiety and depression. Trends Neurosci. 36, 305-312.

Fung, T.C., Olson, C.A., Hsiao, E.Y., 2017. Interactions between the microbiota, immune and nervous systems in health and disease. Nat. Neurosci. 20, 145155.

Goodman, J.C., Robertson, C.S., Grossman, R.G., Narayan, R.K., 1990. Elevation of tumor necrosis factor in head injury. J. Neuroimmunol. 30, 213-217.

Gorle, N., Van Cauwenberghe, C., Libert, C., Vandenbroucke, R.E., 2016. The effect of aging on brain barriers and the consequences for Alzheimer's disease development. Mamm. Genome.

Haesebrouck, F., Pasmans, F., Flahou, B., Chiers, K., Baele, M., Meyns, T., Decostere, A. Ducatelle, R., 2009. Gastric helicobacters in domestic animals and nonhuman primates and their significance for human health. Clin. Microbiol. Rev. 22, 202 223. Table of Contents.

Hu, X., Wang, T., Jin, F., 2016. Alzheimer's disease and gut microbiota. Sci. China Life Sci.

Jiang, H., Hampel, H., Prvulovic, D., Wallin, A., Blennow, K., Li, R., Shen, Y., 2011 Elevated CSF levels of TACE activity and soluble TNF receptors in subjects with mild cognitive impairment and patients with Alzheimer's disease. Mol. Neurodegener. 6, 69.

Johanson, C., Stopa, E., McMillan, P., Roth, D., Funk, J., Krinke, G., 2011. The distributional nexus of choroid plexus to cerebrospinal fluid, ependyma and brain: toxicologic/pathologic phenomena, periventricular destabilization, and lesion spread. Toxicol. Pathol. 39, 186-212.

Johnson, K.S., Ottemann, K.M., 2017. Colonization, localization, and inflammation: the roles of H. pylori chemotaxis in vivo. Curr. Opin. Microbiol. 41, 51-57.

Joosten, M., Blaecher, C., Flahou, B., Ducatelle, R., Haesebrouck, F., Smet, A., 2013. Diversity in bacterium-host interactions within the species Helicobacter heilmannii sensu stricto. Vet. Res. 44, 65.

Kawakubo, M., Ito, Y., Okimura, Y., Kobayashi, M., Sakura, K., Kasama, S., Fukuda, M N., Fukuda, M., Katsuyama, T., Nakayama, J., 2004. Natural antibiotic function of a human gastric mucin against Helicobacter pylori infection. Science 305, $1003-$ 1006.

Kelly, J.R., Minuto, C., Cryan, J.F., Clarke, G., Dinan, T.G., 2017. Cross talk: the microbiota and neurodevelopmental disorders. Front. Neurosci. 11, 490.

Kortekaas, R., Leenders, K.L., van Oostrom, J.C., Vaalburg, W., Bart, J., Willemsen, A.T., Hendrikse, N.H., 2005. Blood-brain barrier dysfunction in parkinsonian midbrain in vivo. Ann. Neurol. 57, 176-179.

Kossmann, T., Hans, V.H., Imhof, H.G., Stocker, R., Grob, P., Trentz, O., MorgantiKossmann, C., 1995. Intrathecal and serum interleukin-6 and the acute-phase response in patients with severe traumatic brain injuries. Shock 4, 311-317.

Kountouras, J., Tsolaki, M., Boziki, M., Gavalas, E., Zavos, C., Stergiopoulos, C., Kapetanakis, N., Chatzopoulos, D., Venizelos, I., 2007. Association between Helicobacter pylori infection and mild cognitive impairment. Eur. J. Neurol. 14, 976-982.

Kufe, D.W., 2009. Mucins in cancer: function, prognosis and therapy. Nat. Rev. Cancer 9, 874-885.

Kunis, G., Baruch, K., Rosenzweig, N., Kertser, A., Miller, O., Berkutzki, T., Schwartz, M., 2013. IFN- $\gamma$-dependent activation of the brain's choroid plexus for CNS immune surveillance and repair. Brain 136, 3427-3440.

Kusters, J.G., van Vliet, A.H., Kuipers, E.J., 2006. Pathogenesis of Helicobacter pylori infection. Clin. Microbiol. Rev. 19, 449-490.

Lee, H., Pienaar, I.S., 2014. Disruption of the blood-brain barrier in Parkinson's disease: curse or route to a cure? Front. Biosci. (Landmark Ed) 19, 272-280.

Li, J., Sung, C.Y., Lee, N., Ni, Y., Pihlajamaki, J., Panagiotou, G., El-Nezami, H., 2016. Probiotics modulated gut microbiota suppresses hepatocellular carcinoma growth in mice. Proc. Natl. Acad. Sci. U.S.A. 113, E1306-E1315.

Li, J.C., Han, L., Wen, Y.X., Yang, Y.X., Li, S., Li, X.S., Zhao, C.J., Wang, T.Y., Chen, H., Liu, Y., Qi, C.L., He, X.D., Gu, Q.L., Ye, Y.X., Zhang, Y., Huang, R., Wu, Y.E., He, R.R. Kurihara, H., Song, X.Y., Cao, L., Wang, L.J., 2015. Increased permeability of the blood-brain barrier and Alzheimer's disease-like alterations in slit-2 transgenic mice. J. Alzheimers Dis. 43, 535-548. 
Liang, J., Ducatelle, R., Pasmans, F., Smet, A., Haesebrouck, F., Flahou, B., 2013. Multilocus sequence typing of the porcine and human gastric pathogen Helicobacter suis. J. Clin. Microbiol. 51, 920-926.

Linden, S., Nordman, H., Hedenbro, J., Hurtig, M., Boren, T., Carlstedt, I., 2002. Strainand blood group-dependent binding of Helicobacter pylori to human gastric MUC5AC glycoforms. Gastroenterology 123, 1923-1930.

Lo, Y.C., Shih, Y.T., Wu, D.C., Lee, Y.C., 2009. In vitro effects of Helicobacter pyloriinduced infection in gastric epithelial AGS cells on microglia-mediated toxicity in neuroblastoma SH-SY5Y cells. Inflamm. Res. 58, 329-335.

Marques, F., Sousa, J.C., 2015. The choroid plexus is modulated by various peripheral stimuli: implications to diseases of the central nervous system. Front. Cell. Neurosci. 9, 136.

Marques, F., Sousa, J.C., Coppola, G., Geschwind, D.H., Sousa, N., Palha, J.A., CorreiaNeves, M., 2009. The choroid plexus response to a repeated peripheral inflammatory stimulus. BMC Neurosci. 10, 135.

Marques, F., Sousa, J.C., Correia-Neves, M., Oliveira, P., Sousa, N., Palha, J.A., 2007. The choroid plexus response to peripheral inflammatory stimulus. Neuroscience 144, 424-430.

Marques, F., Sousa, J.C., Sousa, N., Palha, J.A., 2013. Blood-brain-barriers in aging and in Alzheimer's disease. Mol. Neurodegener. 8, 38.

Mayer, E.A., Knight, R., Mazmanian, S.K., Cryan, J.F., Tillisch, K., 2014. Gut microbes and the brain: paradigm shift in neuroscience. J. Neurosci. 34, 15490-15496.

McGuckin, M.A., Linden, S.K., Sutton, P., Florin, T.H., 2011. Mucin dynamics and enteric pathogens. Nat. Rev. Microbiol. 9, 265-278.

Meeker, R.B., Bragg, D.C., Poulton, W., Hudson, L., 2012. Transmigration of macrophages across the choroid plexus epithelium in response to the feline immunodeficiency virus. Cell Tissue Res. 347, 443-455.

Mridula, K.R., Borgohain, R., Chandrasekhar Reddy, V., Bandaru, V., Suryaprabha, T. 2017. Association of Helicobacter pylori with Parkinson's disease. J. Clin. Neurol. 13, 181-186.

Mulak, A., Bonaz, B., 2015. Brain-gut-microbiota axis in Parkinson's disease. World J. Gastroenterol. 21, 10609-10620.

Nielsen, H.H., Qiu, J., Friis, S., Wermuth, L., Ritz, B., 2012. Treatment for Helicobacter pylori infection and risk of Parkinson's disease in Denmark. Eur. J. Neurol. 19, 864-869.

O’Rourke, J.L., Solnick, J.V., Neilan, B.A., Seidel, K., Hayter, R., Hansen, L.M., Lee, A. 2004. Description of 'Candidatus Helicobacter heilmannii' based on DNA sequence analysis of $16 \mathrm{~S}$ rRNA and urease genes. Int. J. Syst. Evol. Microbiol. 54, 2203-2211.

Ochoa-Reparaz, J., Kasper, L.H., 2014. Gut microbiome and the risk factors in central nervous system autoimmunity. FEBS Lett. 588, 4214-4222.

Parracho, H.M., Bingham, M.O., Gibson, G.R., McCartney, A.L., 2005. Differences between the gut microflora of children with autistic spectrum disorders and that of healthy children. J. Med. Microbiol. 54, 987-991.

Paumier, K.L., Sukoff Rizzo, S.J., Berger, Z., Chen, Y., Gonzales, C., Kaftan, E., Li, L., Lotarski, S., Monaghan, M., Shen, W., Stolyar, P., Vasilyev, D., Zaleska, M. Dunlop, W D.H.J., 2013. Behavioral characterization of A53T mice reveals early and late stage deficits related to Parkinson's disease. PLoS One 8, e70274.

Pere-Vedrenne, C., Flahou, B., Loke, M.F., Menard, A., Vadivelu, J., 2017. Other Helicobacters, gastric and gut microbiota. Helicobacter 22 (Suppl.), 1.

Pisani, V., Stefani, A., Pierantozzi, M., Natoli, S., Stanzione, P., Franciotta, D., Pisani, A., 2012. Increased blood-cerebrospinal fluid transfer of albumin in advanced Parkinson's disease. J. Neuroinflammation 9, 188.

Redzic, Z.B., Preston, J.E., Duncan, J.A., Chodobski, A., Szmydynger-Chodobska, J. 2005. The choroid plexus-cerebrospinal fluid system: from development to aging. Curr. Top. Dev. Biol. 71, 1-52.

Roubaud Baudron, C., Varon, C., Megraud, F., Salles, N., 2016. Alzheimer’s disease and Helicobacter pylori infection: a possible link? Geriatr. Psychol. Neuropsychiatr. Vieil. 14, 86-94.

Schwerk, C., Rybarczyk, K., Essmann, F., Seibt, A., Molleken, M.L., Zeni, P., Schroten, H., Tenenbaum, T., 2010. TNFalpha induces choroid plexus epithelial cell barrier alterations by apoptotic and nonapoptotic mechanisms. J. Biomed. Biotechnol. 2010, 307231.
Shechter, R., London, A., Schwartz, M., 2013. Orchestrated leukocyte recruitment to immune-privileged sites: absolute barriers versus educational gates. Nat. Rev. Immunol. 13, 206-218.

Steffen, B.J., Breier, G., Butcher, E.C., Schulz, M., Engelhardt, B., 1996. ICAM-1, VCAM1, and MAdCAM-1 are expressed on choroid plexus epithelium but not endothelium and mediate binding of lymphocytes in vitro. Am. J. Pathol. 148, 1819-1838.

Stolp, H.B., Dziegielewska, K.M., 2009. Review: role of developmental inflammation and blood-brain barrier dysfunction in neurodevelopmental and neurodegenerative diseases. Neuropathol. Appl. Neurobiol. 35, 132-146.

Strazielle, N., Ghersi-Egea, J.F., 2000. Choroid plexus in the central nervous system: biology and physiopathology. J. Neuropathol. Exp. Neurol. 59, 561-574.

Stridh, L., Ek, C.J., Wang, X., Nilsson, H., Mallard, C., 2013. Regulation of Toll-like receptors in the choroid plexus in the immature brain after systemic inflammatory stimuli. Transl. Stroke Res. 4, 220-227.

Teng, G., Dai, Y., Chu, Y., Li, J., Zhang, H., Wu, T., Shuai, X., Wang, W., 2017. Helicobacter pylori induces CDX2 and COX2 expression by modulating microRNAs in esophageal epithelial cells. Cancer Sci.

Thouvenot, E., Lafon-Cazal, M., Demettre, E., Jouin, P., Bockaert, J., Marin, P., 2006. The proteomic analysis of mouse choroid plexus secretome reveals a high protein secretion capacity of choroidal epithelial cells. Proteomics 6, 59415952.

Tognini, P., 2017. Gut microbiota: a potential regulator of neurodevelopment. Front. Cell. Neurosci. 11, 25.

Van Hauwermeiren, F., Vandenbroucke, R.E., Grine, L., Lodens, S., Van Wonterghem, E., De Rycke, R., De Geest, N., Hassan, B., Libert, C., 2015. TNFR1-induced lethal inflammation is mediated by goblet and Paneth cell dysfunction. Mucosal Immunol. 8, 828-840.

Vandenbroucke, R.E., 2016. A hidden epithelial barrier in the brain with a central role in regulating brain homeostasis. Implications for aging. Ann. Am. Thorac. Soc. 13 , S407-S410.

Vandenbroucke, R.E., Dejonckheere, E., Van Lint, P., Demeestere, D., Van Wonterghem, E., Vanlaere, I., Puimege, L., Van Hauwermeiren, F., De Rycke, R., Mc Guire, C., Campestre, C., Lopez-Otin, C., Matthys, P., Leclercq, G., Libert, C., 2012. Matrix metalloprotease 8-dependent extracellular matrix cleavage at the blood-CSF barrier contributes to lethality during systemic inflammatory diseases. J. Neurosci. 32, 9805-9816.

Vandenbroucke, R.E., Vanlaere, I., Van Hauwermeiren, F., Van Wonterghem, E., Wilson, C., Libert, C., 2014. Pro-inflammatory effects of matrix metalloproteinase 7 in acute inflammation. Mucosal Immunol. 7, 579-588.

Varatharaj, A., Galea, I., 2016. The blood-brain barrier in systemic inflammation. Brain Behav. Immun.

Vawter, M.P., Dillon-Carter, O., Tourtellotte, W.W., Carvey, P., Freed, W.J., 1996 TGFbeta1 and TGFbeta2 concentrations are elevated in Parkinson's disease in ventricular cerebrospinal fluid. Exp. Neurol. 142, 313-322.

Walters, W.A., Xu, Z., Knight, R., 2014. Meta-analyses of human gut microbes associated with obesity and IBD. FEBS Lett. 588, 4223-4233.

Wirdefeldt, K., Adami, H.O., Cole, P., Trichopoulos, D., Mandel, J., 2011. Epidemiology and etiology of Parkinson's disease: a review of the evidence. Eur. J. Epidemiol. 26 (Suppl. 1), S1-S58.

Wong, F., Rayner-Hartley, E., Byrne, M.F., 2014. Extraintestinal manifestations of Helicobacter pylori: a concise review. World J. Gastroenterol. 20, 11950-11961.

Wroblewski, L.E., Peek Jr., R.M., 2011. Targeted disruption of the epithelial-barrier by Helicobacter pylori. Cell Commun. Signal. 9, 29.

Yamashiro, S., 2006. Dellmann's textbook of veterinary histology. Can. Vet. J. 48, 414.

Zeni, P., Doepker, E., Schulze-Topphoff, U., Huewel, S., Tenenbaum, T., Galla, H.J., 2007. MMPs contribute to TNF-alpha-induced alteration of the bloodcerebrospinal fluid barrier in vitro. Am. J. Physiol. Cell Physiol. 293, C855-C864.

Zhang, Y.J., Li, S., Gan, R.Y., Zhou, T., Xu, D.P., Li, H.B., 2015. Impacts of gut bacteria on human health and diseases. Int. J. Mol. Sci. 16, 7493-7519. 